\title{
Lectures on the Near-Side Ridge, Landau Hydrodynamics, and Heavy Quarkonia in High Energy Heavy-Ion Collisions
}

\author{
Cheuk-Yin Wong \\ Oak Ridge National Laboratory, Oak Ridge, USA
}

\begin{abstract}
We give an introduction to three different topics that are of current interest in heavy-ion collisions. Particles associated with the near-side jet are found to exhibit a $\Delta \phi-\Delta \eta$ correlation in the form of a ridge in the $\Delta \eta$ direction but a peak at $\Delta \phi \sim 0$. The experimental data support the description that the ridge particles are medium partons kicked by the jet. The measurement of the characteristics of the ridge provides a unique tool to probe the nature of the (jet parton)-(medium parton) collision and the momentum distribution of dense matter formed in the early stage of the heavy-ion collision. We find that the magnitude of the longitudinal momentum kick along the jet direction acquired by a medium parton in collision with the jet is about $1 \mathrm{GeV}$, and the early parton momentum distribution is in the form of a rapidity plateau with a thermal-type transverse momentum distribution. In the second lecture, we re-examine the validity of Landau hydrodynamics which provides a reasonable description of the space-time dynamics of the hot matter produced in high-energy heavy-ion collisions. We find that the rapidity distribution of produced particles should be more appropriately modified from Landau's result. Past successes of the Gaussian distribution in explaining experimental rapidity data can be understood, not because it is an approximation of the original Landau distribution, but because it is in fact a close representation of the modified distribution. In the final lecture, we give an introduction to the development of the potential model for quarkonia, using thermodynamical quantities obtained in lattice gauge calculations. We find that the potential model is consistent with the lattice gauge spectral function analysis, if the color-singlet heavy quark-antiquark potential is a linear combination of the color-singlet free energy $F_{1}$ and internal energy $U_{1}$, with coefficients that depend on the equation of state. We find that the effects of dynamical quark modifies only slightly the stability of $J / \psi$ and the quark drip line limits possible quarkonium states with light quarks to temperatures close to, and slightly greater than, the critical phase transition temperature.
\end{abstract}

The research and the participation at this summer school was supported in part by the HISS DNA program of the Helmholtz Association and by the Division of Nuclear Physics, U.S. Department of Energy, under Contract No. DE-AC05-00OR22725, managed by UT-Battelle, LLC.

This is an Open Access article distributed under the terms of the Creative Commons Attribution-Noncommercial License 3.0, which permits unrestricted use, distribution, and reproduction in any noncommercial medium, provided the original work is properly cited. 


\section{Near-Side Ridge and Early Parton Momentum \\ Distribution \\ Cheuk-Yin Wong \\ Oak Ridge National Laboratory}

- Introduction

- the jet, near-side jet, away-side jet, and the ridge

- how does the ridge arise

- The relation between the early parton momentum distribution \& the ridge distribution

- Use the experimental ridge distribution to extract the early parton momentum distribution

- Conclusions

C.Y.Wong, Phy.Rev.C76,054908('07)

C.Y.Wong, Chin.Phys.Lett.25,3936('08)

C.Y.Wong, J.Phys.G35,104085('08)

C.Y.Wong, Phys. Rev.C78,064905('08)

C.Y.Wong, arXiv:0901.0726(PRC,in press)

C.Y.Wong, arXiv:0903.3879('09)

\section{Why study the early parton momentum distribution?}

- Early parton momentum distribution is an important quantity

- It provides information in early collision dynamics

- It provides initial value data for subsequent evolution to quark-gluon plasma

- Not much is known experimentally about early parton momentum distribution

- We propose to use the ridge distribution to extract early parton momentum distribution 


\section{Jet production}

Jets are particles with high transverse momenta produced by nucleon-nucleon hard-scattering process.

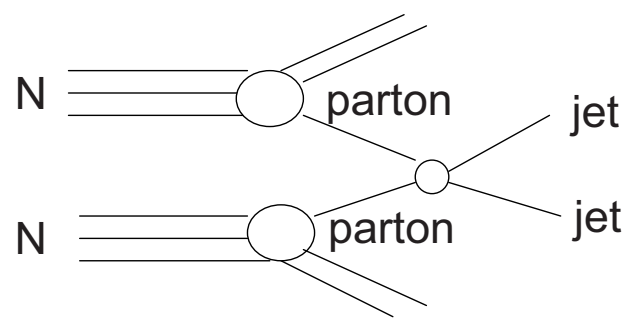

\section{Jet properties}

- Jets are particles with high transverse momenta.

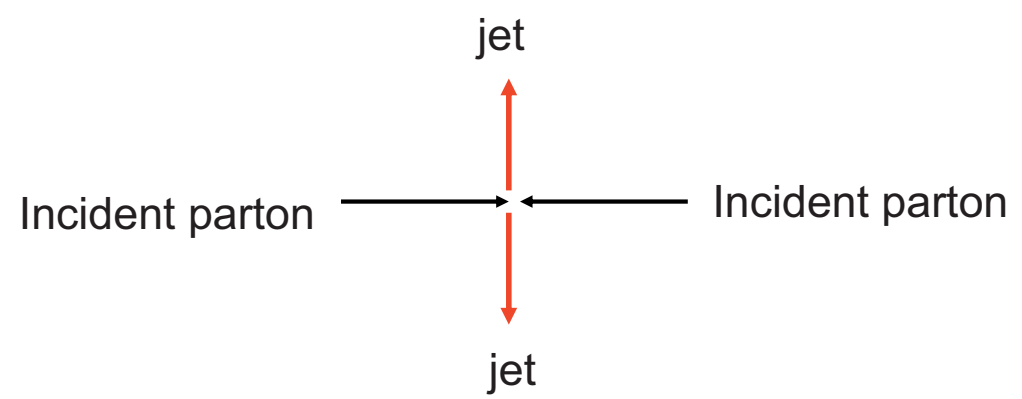

- Jets come as a back-to-back pair

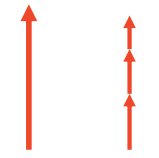

-A jet is nearly degenerate with its fragments

jet fragments

- The jet fragments form a jet cone 
The term "jet" are often used for different objects:

- jet parton (the totality of all jet fragments)

- $\quad$ trigger particle (one of the jet fragments)

- fragments in the jet cone (not including the trigger)

Ambiguity can be removed by judging from the context.

\section{Near-side and away-side jets}

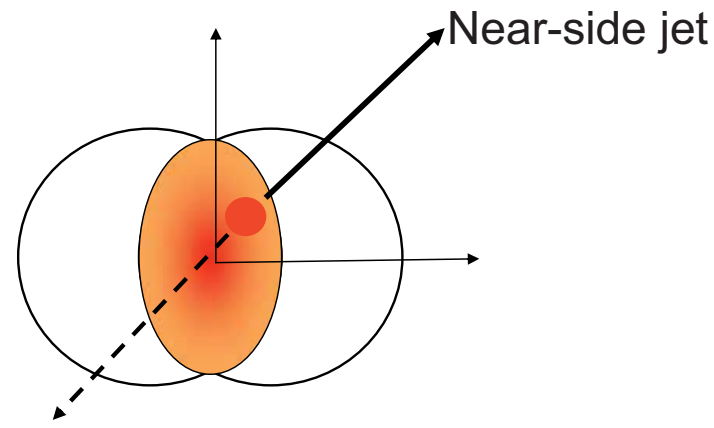

Away-side jet

Away-side jet is subject to strong absorption.

Absorption is strongest in the most-central collision 


\section{What is the ridge phenomenon? \\ - Occurrence of a near-side "jet" \\ - Associated particles are observed in coincidence with the jet \\ - The momentum and angles of these particles are measured: \\ pt(particle)

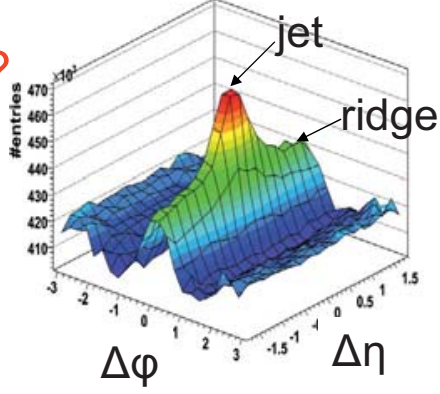 \\ $\Delta \varphi=\varphi$ (particle) $-\varphi$ (jet) \\ $\Delta \eta=\eta$ (particle) $-\varphi$ (jet)}

Find: $\Delta \varphi-\Delta \eta$ correlation

Probability distribution $\mathrm{P}(\Delta \varphi, \Delta \eta)$ in $\Delta \varphi-\Delta \eta$ is in the form of

(i) a "jet component"

(ii) a "ridge component".

\section{Experimental information about the ridge}

- (i) Ridge yield correlated with N_participants

- (ii) Ridge yield nearly independent of pt, flavor, baryon, meson characters of the jet trigger

- (iii) Tjet $>>$ Tridge $>$ Tinclusive

- (iv) Ridge particles have $\Delta \varphi \sim 0$

- (v) Ridge particles nearly uniform in $\Delta \eta$ 


\section{Many Ridge Models}

- C.Y.Wong,Phy.Rev.C76,054908('07);Chin.Phys.Lett.25,3936('08);Phys.Rev.C78,064 905('08);J. Phys.G35,104085('08);arXiv:0901.0726(PRC,in press);arXiv:0903.3879.

- S.A.Voloshin, Phys. Lett. B632, 490 ('06)

- E. Shuryak, Phys.Rev.C76, 047901 ('07)

- V. S. Pantuev, arxiv:0710.1882('07)

- C. B. Chiu and R.C. Hwa, Phys. Rev. C76, 047901 ('08)

- N. Armesto, C. A. Salgado, U. A. Wiedemann, Phys.Rev.C76,054908('07)

- A.Dumitru, Y.Nara,B.Schenke,M.Strickland, Phys.Rev.C78,024909('08)

- A.majumder,B.Mueller, and S.A.Bass, Phys. Rev. Lett. 99, 042301 ('07)

- R.Mizukawa, T.Hirano,M.Isse, Y.Nara,A.Ohnishi, J.Phys.G35,104083('08)

- S.Gavin,L.McLerran, G.Moschelle, arXiv:0806.4718

- A. Dumitru,F. Gelis, L. McLerran, and R. Venugoplan, Nucl . Phys . A810, 91 (' 09)

- -.. many more

\section{Questions}

- How does the ridge phenomenon occur?

- What is the momentum distribution of the early medium partons?

- What is the dominant mechanism of jet momentum loss?

These questions are linked together and can be answered by the momentum kick model:

Ridge particles are medium partons kicked by the jet. The kicked partons carry direct information on the medium parton momentum distribution and the magnitude of the momentum kick. The momentum kick is related to the jet momentum loss. 


\section{Schematic picture of the momentum kick model}

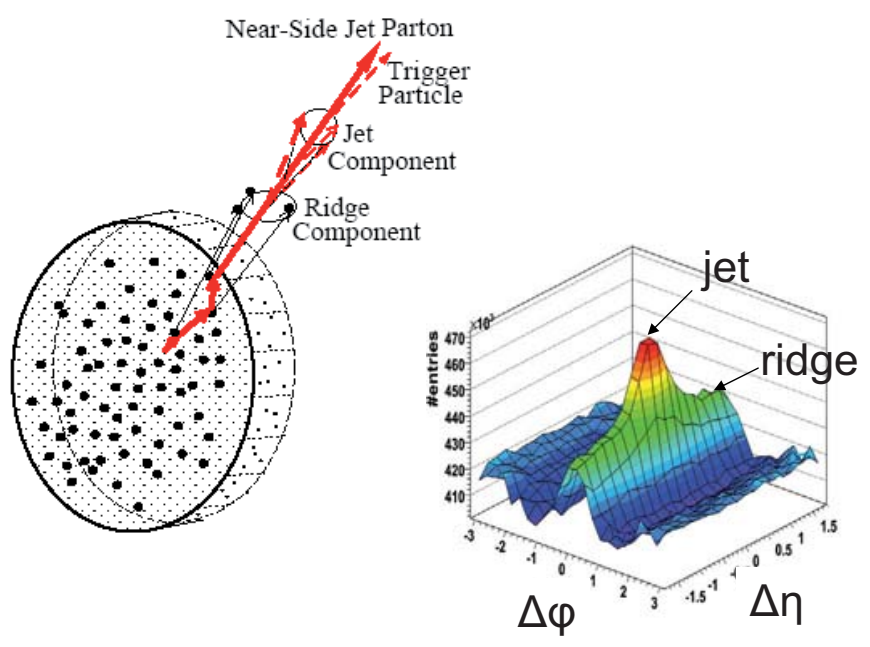

Ridge particles are medium partons kicked by the jet

- (i) Ridge yield correlated with N_participants

- (ii) Ridge yield nearly independent of pt trigger, flavor, baryon, meson characters of the jet

- (iii) $T_{\text {jet }}>>T_{\text {ridge }} \geq T_{\text {inclusive }}$

- (iv) $\Delta \varphi \sim 0$ implies that the ridge particles acquire their azimuthally properties from the jet

- (v) jet-(medium parton) interactions are short-ranged because of non-perturbative screening

The most likely explanation:

ridge particles are medium partons kicked by the jet and they acquire a momentum kick q along the jet direction 


\section{Basic idea of the momentum kick model}

Normalized initialmedium parton momentum distribution $d F / d \vec{p}_{i}$

$$
\begin{aligned}
& \text { before kick }=\frac{d F}{d \vec{p}_{i}}\left(\vec{p}_{i}\right), \quad \int d \vec{p}_{i} \frac{d F}{d \vec{p}_{i}}\left(\vec{p}_{i}\right)=1 \\
& \text { after kick }=\left[\frac{d F}{d \vec{p}_{i}}\left(\vec{p}_{i}\right)\right]_{\vec{p}_{i}=\vec{p}_{f}-\vec{q}}
\end{aligned}
$$

A single jet kicks $\langle N\rangle$ medium partons

Ridge particle momentum distribution $=\langle N\rangle\left[\frac{d F}{d \vec{p}_{i}}\left(\vec{p}_{i}\right)\right] \vec{p}_{i}=\vec{p}_{f}-\vec{q}$

We observed only charge hadrons,

$$
\frac{d N_{\text {ridge }}^{A A}}{d \vec{p}_{f}}\left(\vec{p}_{f}\right) \text { per trigger }=\frac{2}{3}\langle N\rangle\left[\frac{d F}{d \vec{p}_{i}}\left(\vec{p}_{i}\right)\right] \vec{p}_{i}=\vec{p}_{f}-\vec{q}
$$

A ridge particle is attenuated by a factor $f_{R}$

$$
\frac{d N_{\text {ridge }}^{A A}}{N_{\text {trig }} d \vec{p}_{f}}\left(\vec{p}_{f}\right)=\frac{2}{3} f_{R}\langle N\rangle\left[\frac{d F}{d \vec{p}_{i}}\left(\vec{p}_{i}\right)\right]_{\vec{p}_{i}=\vec{p}_{f}-\vec{q}}
$$

- Ridge yield per trigger depends on the number of kicked medium particles, $<\mathrm{N}>$. 


\section{Mathematical details}

trigger yield in pp $=\frac{d N^{p p}}{d \vec{p}_{\text {trig }}}\left(\vec{p}_{\text {trig }}\right)=\int d \vec{p}_{j} \frac{d N_{j}^{p p}}{d \vec{p}_{j}} \widetilde{D}\left(\vec{p}_{\text {trig }}, \vec{p}_{j}\right)$

We introduce $D\left(\vec{p}_{\text {trig }}, \vec{p}_{j}\right)$,

$$
\begin{aligned}
1 & =\int d \vec{p}_{j} \frac{d N_{j}^{p p}}{d \vec{p}_{j}} D\left(\vec{p}_{t r i g}, \vec{p}_{j}\right) \\
D\left(\vec{p}_{t r i g}, \vec{p}_{j}\right) & =\widetilde{D}\left(\vec{p}_{t r i g}, \vec{p}_{j}\right) / \frac{d N^{p p}}{d \vec{p}_{t r i g}}\left(\vec{p}_{t r i g}\right) .
\end{aligned}
$$

jet source in an AA collision :

$$
\begin{gathered}
\frac{d N_{j}^{A A}}{d \vec{p}_{j}}\left(\vec{p}_{j}\right)=\sum_{i=1}^{N b i n} \frac{d N_{j}^{i(p p)}}{d \vec{p}_{j}}\left(\vec{p}_{j}\right) \\
N_{\text {bin }}=\int d \vec{p}_{j} \frac{d N_{j}^{A A}}{d \vec{p}_{j}}\left(\vec{p}_{j}\right) D\left(\vec{p}_{\text {trig }}, \vec{p}_{j}\right) \\
N_{\text {trig }}=\int d \vec{p}_{j} \frac{d N_{j}^{A A}}{d \vec{p}_{j}} \sum_{N=0}^{N \max } P(N) e^{-\zeta_{a} N} D\left(\vec{p}_{\text {trig }}, \vec{p}_{j}-\sum_{n=1}^{N} \vec{q}_{n}-\vec{\Delta}_{r}\right) \\
R_{A A}=\frac{1}{N_{\text {bin }}} \int d \vec{p}_{j} \frac{d N_{j}^{A A}}{d \vec{p}_{j}} \sum_{N=0}^{N \max } P(N) e^{-\zeta_{a} N} D\left(\vec{p}_{t r i g}, \vec{p}_{j}-\sum_{n=1}^{N} \vec{q}_{n}-\vec{\Delta}_{r}\right)
\end{gathered}
$$

\section{Simplification for $\mathrm{N}_{\text {trig }}$ and $\mathrm{R}_{\mathrm{AA}}$}

$$
\begin{aligned}
& N_{\text {trig }}=\int d \vec{p}_{j} \frac{d N_{j}^{A A}}{d \vec{p}_{j}} \sum_{N=0}^{N \max } P(N) e^{-\zeta_{a} N} D\left(\vec{p}_{\text {trig }}, \vec{p}_{j}-\sum_{n=1}^{N} \vec{q}_{n}-\vec{\Delta}_{r}\right) \\
& R_{A A}=\frac{1}{N_{b i n}} \int d \vec{p}_{j} \frac{d N_{j}^{A A}}{d \vec{p}_{j}} \sum_{N=0}^{N \max } P(N) e^{-\zeta_{a} N} D\left(\vec{p}_{\text {trig }}, \vec{p}_{j}-\sum_{n=1}^{N} \vec{q}_{n}-\vec{\Delta}_{r}\right)
\end{aligned}
$$

The fragmentation function can be written approximately as

$$
D\left(\vec{p}_{\text {trig }}, \vec{p}_{j}-\sum_{n=1}^{N} \vec{q}_{n}-\vec{\Delta}_{r}\right)=e^{-\left(\zeta_{f}+\zeta_{r}\right) N} D\left(\vec{p}_{\text {trig }}, \vec{p}_{j}\right)
$$

Then

$$
\begin{aligned}
& N_{\text {trig }}=\int d \vec{p}_{j} \frac{d N_{j}^{A A}}{d \vec{p}_{j}} D\left(\vec{p}_{\text {trig }}, \vec{p}_{j}\right) \sum_{N=0}^{N \max } P(N) e^{-\left(\zeta_{a}+\zeta_{f}+\zeta_{r}\right) N} \\
& N_{\text {trig }}=N_{\text {bin }} \sum_{N=0}^{N \max } P(N) e^{-\zeta N} ; \quad \zeta=\zeta_{a}+\zeta_{f}+\zeta_{r} . \\
& R_{A A}=\sum_{N=0}^{N \max } P(N) e^{-\zeta N}
\end{aligned}
$$




\section{How to calculate the ridge yield per trigger?}

$$
\begin{aligned}
\frac{d N_{\text {ridge }}^{A A}}{d \vec{p}}=\int d \vec{p}_{j} \frac{d N_{j}^{A A}}{d \vec{p}_{j}} \sum_{N=0}^{N_{\max }} P(N) & e^{-\zeta_{a}^{N}} D\left(\vec{p}_{\text {trig }}, \vec{p}_{j}-\sum_{n=1}^{N} \vec{q}_{n}-\vec{\Delta}_{r}\right) \\
\times & \frac{2}{3} \sum_{n=1}^{N}\left\{f_{R n} \frac{d F_{n}}{d \vec{p}}\left(\vec{q}_{n}\right)\right\}
\end{aligned}
$$

Result implified by using the average ridge yeild per kick

$$
\begin{aligned}
& \frac{d N_{\text {ridge }}^{A A}}{d \vec{p}}=\frac{2}{3} f_{R} \frac{d F}{d \vec{p}}(\vec{q}) \\
& \times \int d \vec{p}_{j} \frac{d N_{j}^{A A}}{d \vec{p}_{j}} \sum_{N=0}^{N_{\max }} N P(N) e^{-\zeta_{a} N} D\left(\vec{p}_{\text {trig }}, \vec{p}_{j}-\sum_{n=1}^{N} \vec{q}_{n}-\vec{\Delta}_{r}\right) \\
& \frac{d N_{\text {ridge }}^{A A}}{N_{\text {trig }} d \vec{p}}=\frac{2}{3} f_{R}\langle N\rangle \frac{d F}{d \vec{p}}(\vec{q}) \\
&\langle N\rangle=\frac{\int d \vec{p}_{j} \frac{d N_{j}^{A A}}{d \vec{p}_{j}} \sum_{N=0}^{N \max } N P(N) e^{-\zeta_{s} N} D\left(\vec{p}_{\text {trig }}, \vec{p}_{j}-\sum_{n=1}^{N} \vec{q}_{n}-\vec{\Delta}_{r}\right)}{\int d \vec{p}_{j} \frac{d N_{j}^{A A}}{d \vec{p}_{j}} \sum_{N=0}^{N \max } P(N) e^{-\zeta_{a} N} D\left(\vec{p}_{\text {trig }}, \vec{p}_{j}-\sum_{n=1}^{N} \vec{q}_{n}-\vec{\Delta}_{r}\right)}
\end{aligned}
$$

\section{Relation between the ridge distribution and the early parton momentum distribution}

The normalized ridge particle distribution in the collider frame:

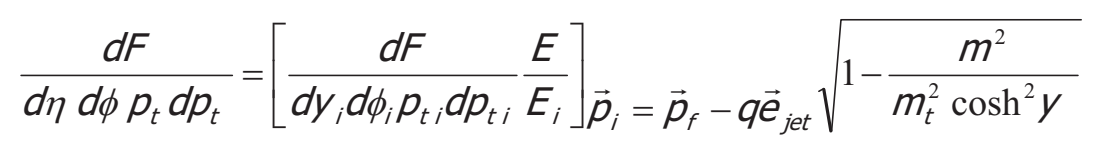

-The kicked final partons subsequently materialize as hadrons by parton-hadron duality

-The ridge particle distribution depends on the initial parton momentum distribution and the momentum kick q. 


\section{Parametrization of initial parton momentum distribution}

We parametrize the normalized initial parton distribution by

$$
\begin{aligned}
\frac{d F}{d y d \phi p_{t} d p_{t}} & =A_{i}(1-x)^{a} \frac{\exp \left\{-\left(\sqrt{m^{2}+p_{t}^{2}}-m\right) / T\right\}}{\sqrt{m_{d}^{2}+p_{t}^{2}}} \\
x & =\frac{\left(p_{0}+p_{z}\right)_{\text {parton }}}{\left(p_{0}+p_{z}\right)_{\text {parent }}}=\frac{\sqrt{m^{2}+p_{t}^{2}}}{m} \exp \left\{|y|-y_{b}\right\} \leq 1
\end{aligned}
$$

$A_{i}$ is a normalization constant such that $\int d \vec{p} d F / d \vec{p}=1$.

The parameters are :

$$
a, T, m_{d}, m \text {, and } y_{b}
$$

We assume parton - hadron duality and take $m=m_{\text {parton }}=m_{\text {pion }}$

$$
y_{b}=y_{\text {beam proton }}
$$

\section{Ridge yield is a maximum at $\Delta \varphi \sim 0$}

For the same magnitude of $\left|\vec{p}_{t f}\right|$ but different $\Delta \phi$, the magnitude of $\left|\vec{p}_{t i}\right|$ is smallest when $\Delta \phi=0$.

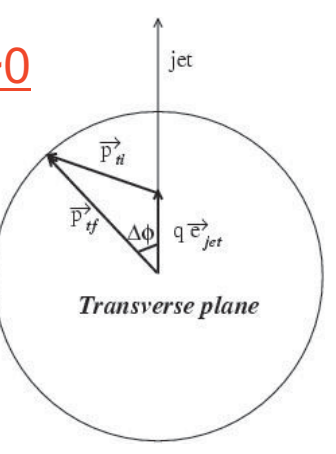

The smaller the magnitude of initial transverse momentum $\left|\vec{p}_{t i}\right|$, the greater the probility of its occurence.

Therefore, the ridge particle yield is a maximum at $\Delta \phi \approx 0$. 


\section{The width in $\Delta \varphi$ depends on the magnitude of $\mathrm{q}$.}

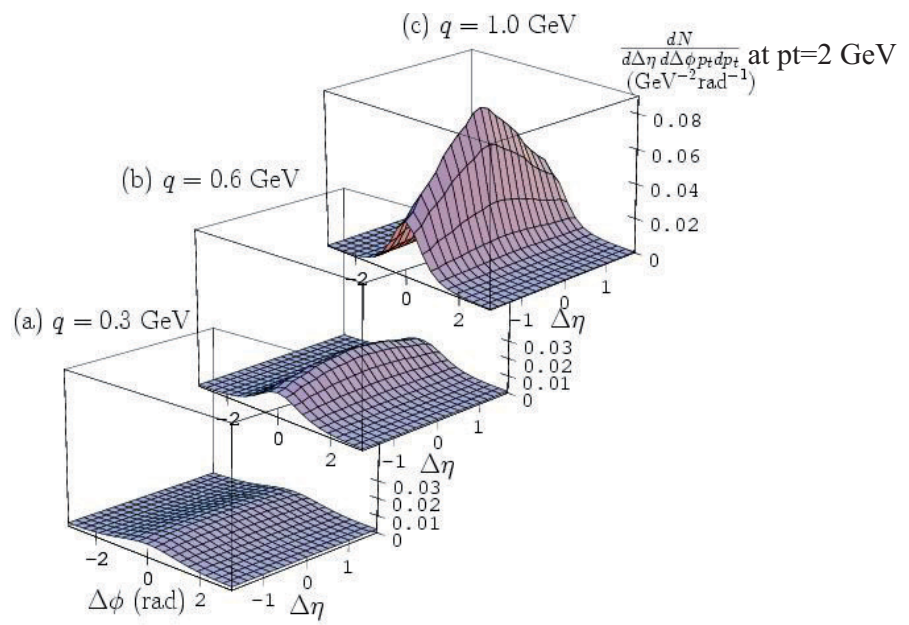

\section{The ridge shape in $\Delta \eta$ initial parton $\mathrm{dN} / \mathrm{dy} \sim(1-\mathrm{x})^{\mathrm{a}}$}

Boost invariant distribution corresponds to $\mathrm{a}=0$.

The shape in $\Delta \eta$ around $\Delta \eta=0$ depends weakly on a.

The shape in $\Delta \eta$ around large $\Delta \eta$ depends strongly on a. 


\section{Experimnetal measurement contains both jet and} $\underline{\text { ridge components }}$

- We need to get a good description of the "jet" component

- The jet component is produced mainly by fragmentation outside the medium. It is the same as in pp collisions for high pt jet.

- We assume that the jet component is an attenuated pp

(jet component in $A A)=f_{J}$ (jet component in $p p$ )

- We need to parameterize pp jet data.

The observed distribution in the momentum kick model

$$
\begin{aligned}
\left.\frac{d N_{c h}}{d \eta d \phi p_{t} d p_{t}}\right|_{A A, \text { total }} ^{\text {observed }}= & \left.\frac{2}{3} f_{R}\langle N\rangle \frac{d F}{d \eta_{f} d \phi_{f} p_{t f} d p_{t f}}\right|_{A A, \text { ridge }} \\
& +\left.f_{J} \frac{d N_{j e t}}{d \eta_{f} d \phi_{f} p_{t f} d p_{t f}}\right|_{p p, \text { jet }}
\end{aligned}
$$

$f_{R}$ and $f_{J}$ are the attenuation factors

we take $f_{J}=\int_{0}^{\lambda} \exp \{-X / \lambda\} d x / \int_{0}^{\lambda} d x=\frac{1-e^{-1}}{1}=0.632$

We need the pp near-side jet data 


\section{The pp near-side jet data can be described by}

$$
\begin{aligned}
& \left.\frac{d N_{j e t}}{d y d \phi p_{t} d p_{t}}\right|_{\mathrm{pp}}=N_{j e t} A_{j e t} \exp \left\{-\sqrt{m^{2}+p_{t}^{2}} / T_{j e t}\right\} \frac{\exp \left\{-\left((\Delta \varphi)^{2}+(\Delta \eta)^{2}\right) / 2 \sigma^{2}\right\}}{2 \pi \sigma^{2}} \\
& N_{\text {jet }}=0.75, \quad A_{\text {jet }}=\frac{\exp \left\{m / T_{\text {jet }}\right\}}{T_{\text {jet }}\left(m+T_{\text {jet }}\right)} \text { is a normalization constant, } \\
& T_{\text {jet }}=0.55 \mathrm{GeV} \text {, } \\
& \sigma=\sigma_{0} \frac{m_{a}}{\sqrt{m_{a}^{2}+p_{t}^{2}}}, \quad \sigma_{0}=0.5, \quad m_{a}=1.1 \mathrm{GeV}
\end{aligned}
$$

pp near-side jet data (open blue circles)
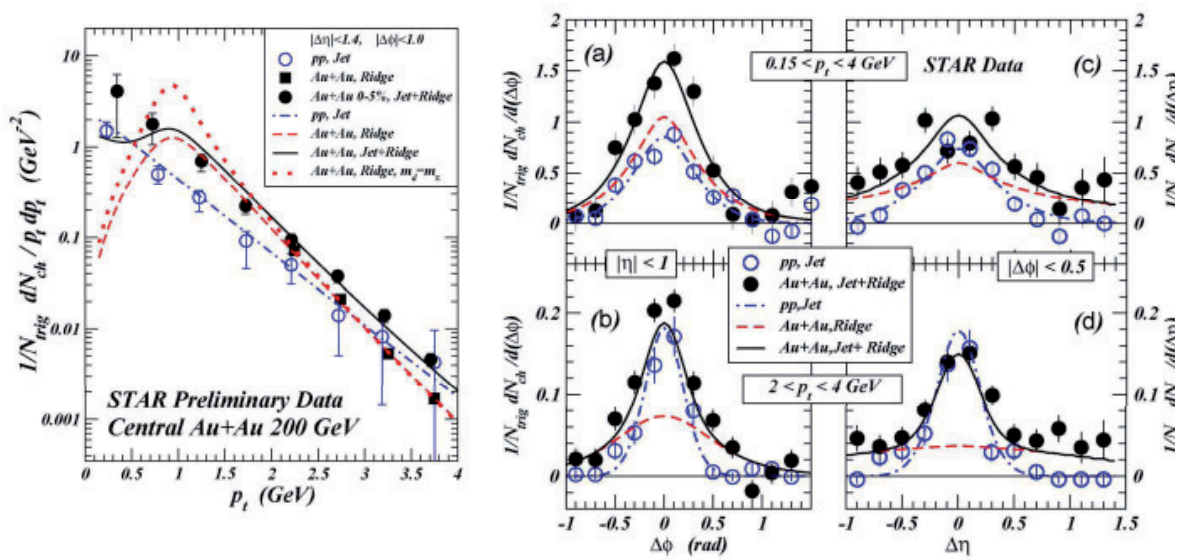

Data from

PRL95,152301(05) \& J. Phy. G34, S679 (07) 


\section{The initial parton momentum distribution}

$$
\begin{gathered}
\frac{d F}{d y d \phi p_{t} d p_{t}}=A_{i}(1-x)^{a} \frac{\exp \left\{-\left(\sqrt{m^{2}+p_{t}^{2}}-m\right) / T\right\}}{\sqrt{m_{d}^{2}+p_{t}^{2}}} \\
x=\frac{\sqrt{m^{2}+p_{t}^{2}}}{m_{b}} \exp \left\{|y|-y_{b}\right\} \leq 1
\end{gathered}
$$

Assuming $m_{b}=m_{\pi}=m, y_{b}=y_{\text {beam }}$, we find

$$
q=1.0 \mathrm{GeV}, \frac{2}{3} f_{R}\langle N\rangle=2.5, \quad a=0.5, T=0.5 \mathrm{GeV}, m_{d}=1 \mathrm{GeV} .
$$

AA near-side data (black solid points) described well by the momentum kick model around $\Delta \eta \sim 0$
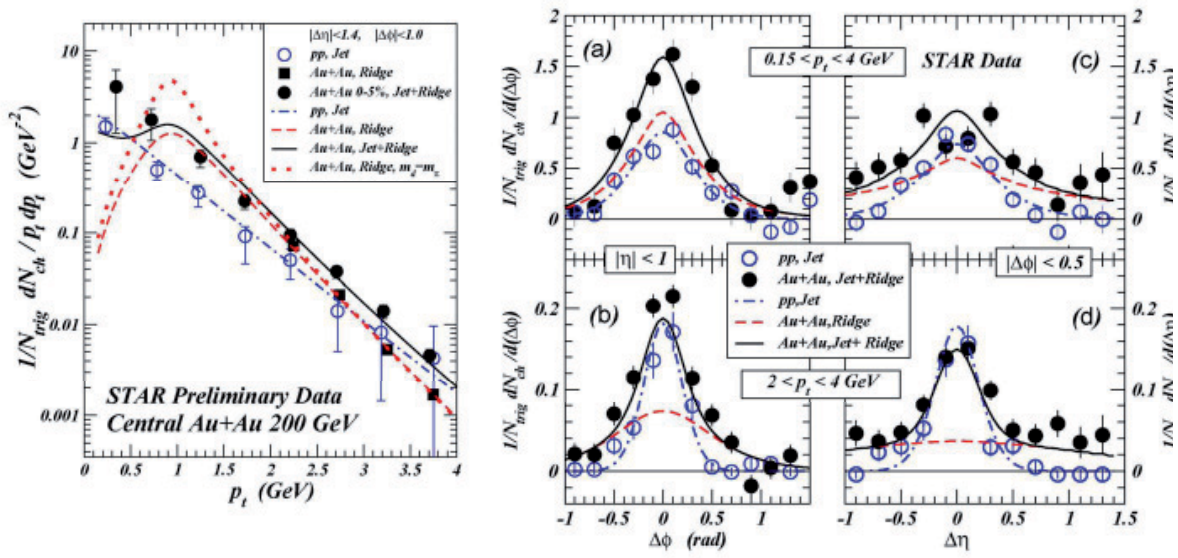

Data from STAR Collaboration

PRL95,152301(05) \& J. Phy. G34, S679 (07) 
AA near-side data (black solid points) described well by the momentum kick model around $|\Delta \eta| \sim 3.3$

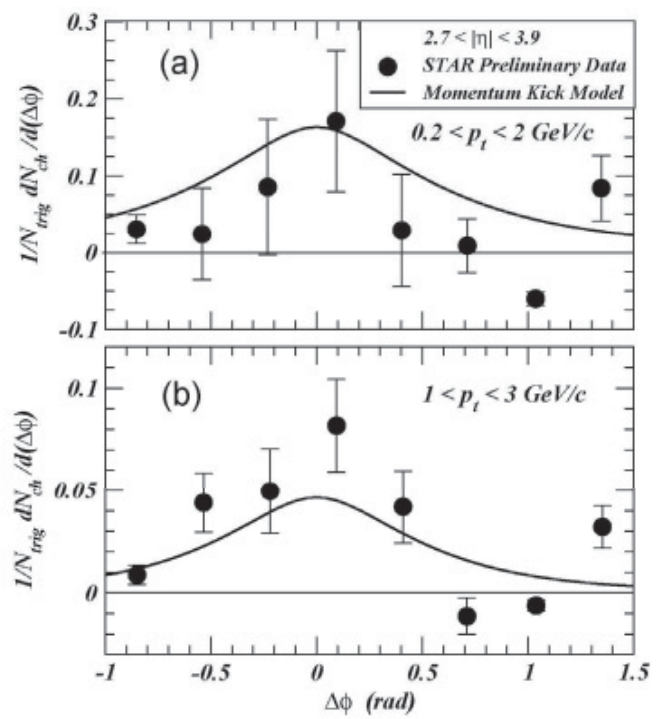

Data from STAR Col. F. Wang et al. arXiv:0707.0815 ('07)

Momentum kick model gives the correct prediction

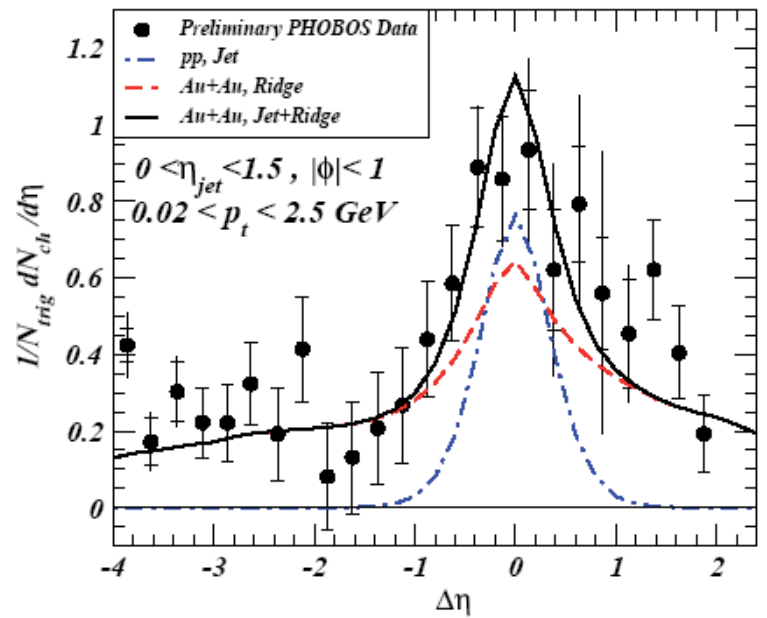




\section{Shape of early parton momentum distribution}

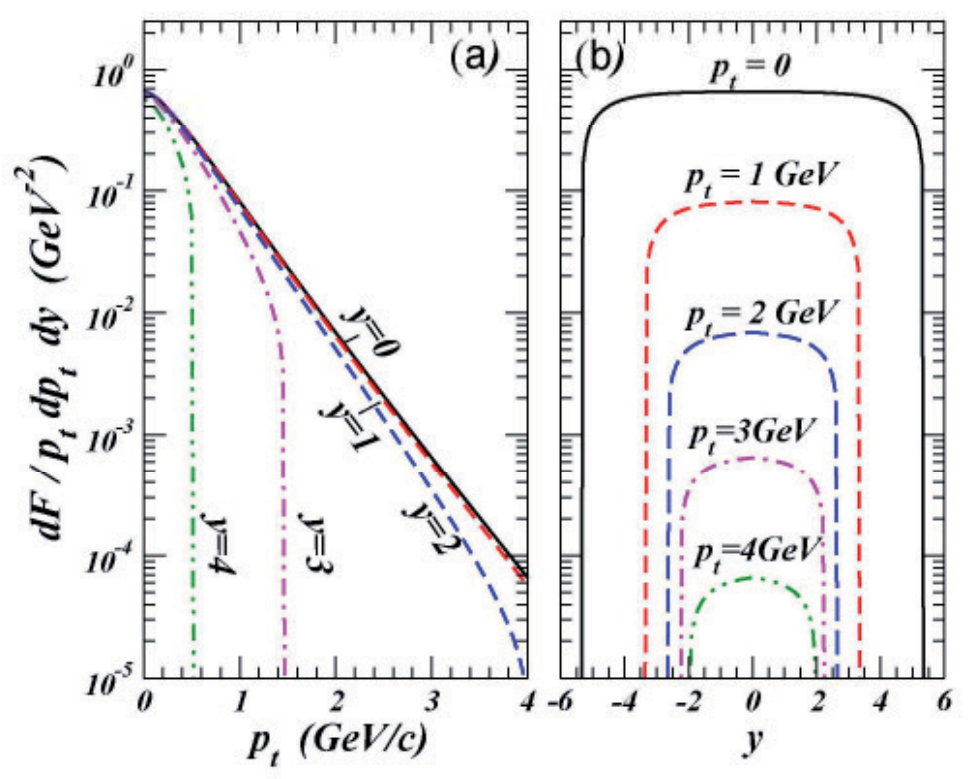

\section{$\underline{\text { Possible evolution scenario of medium partons }}$}
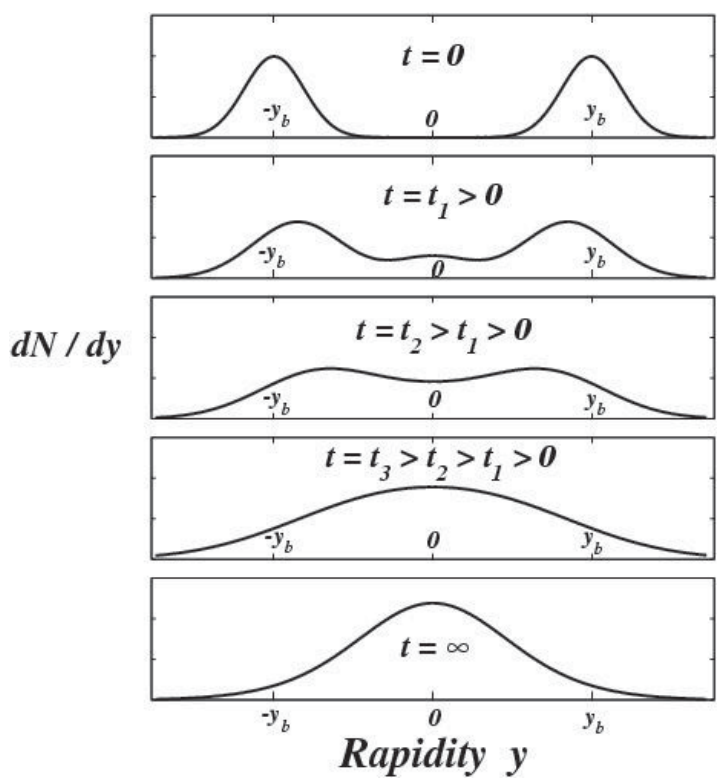


\section{$\underline{\text { Conclusions }}$}

- The ridge particles can be described as medium partons kicked by the jet, and they carry information on the early parton momentum distribution and the momentum kick.

- The parton momentum distribution at the moment of jet-parton collision is relatively flat in rapidity with a thermal-like transverse momentum distribution and sharp kinematic boundaries.

- The magnitude of the momentum kick gained by the parton is $1 \mathrm{GeV}$, which is also the momentum loss by the jet in a jet-parton collision.

\section{Landau Hydrodynamics \\ Cheuk-Yin Wong \\ Oak Ridge National Laboratory}

- Introduction

- Landau hydrodynamics

-- predictions on total $\mathrm{N}$

-- prediction on differential $\mathrm{dN} / \mathrm{dy}$

-- space-time dynamics

- Modification of Landau's dN/dy

- Comparison with experiment

- Conclusions 


\section{Why study Landau hydrodynamics?}

- L.D. Landau, Izv. Akad. Nauk SSSR, 17, 51 (1953)

- Belenkij and L.D.Landau,Usp.Fiz.Nauk. 56, 309 (1955)

- It gives a good description of experimental data

- It gives a simple description of the space-time dynamics of the dense hot matter produced in heavy-ion collisions

- Dense hot matter evolution is needed in many problems

- It is very simple

\section{Recent revived interest in Landau hydrodynamics}

- BRAHM dN/dy data agree with Landau hydrodynamics

$$
\begin{aligned}
& \frac{d N}{d y}=K s^{1 / 4} \frac{1}{\sqrt{2 \pi L}} \exp \left\{-\frac{y^{2}}{2 L}\right\} \\
& L=\ln \left(\frac{\sqrt{S_{N N}}}{2 m_{p}}\right)
\end{aligned}
$$

$\mathrm{L}$ is the logarithm of the Lorentz contraction factor

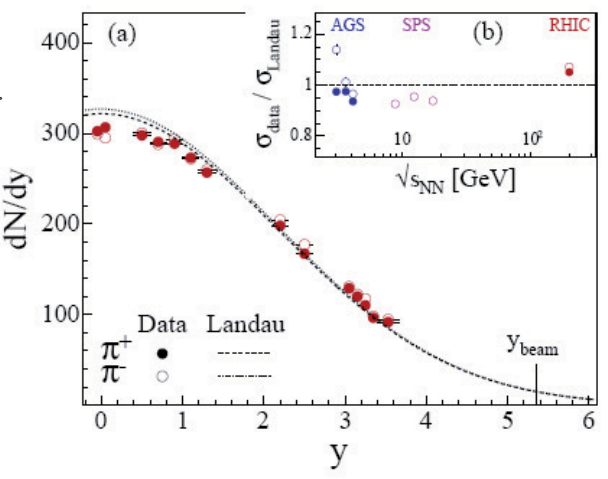

Murray, J. Phys. G30, S667 (2004) 


\section{Landau hydrodynamics give the correct multiplicity in AA collisions}

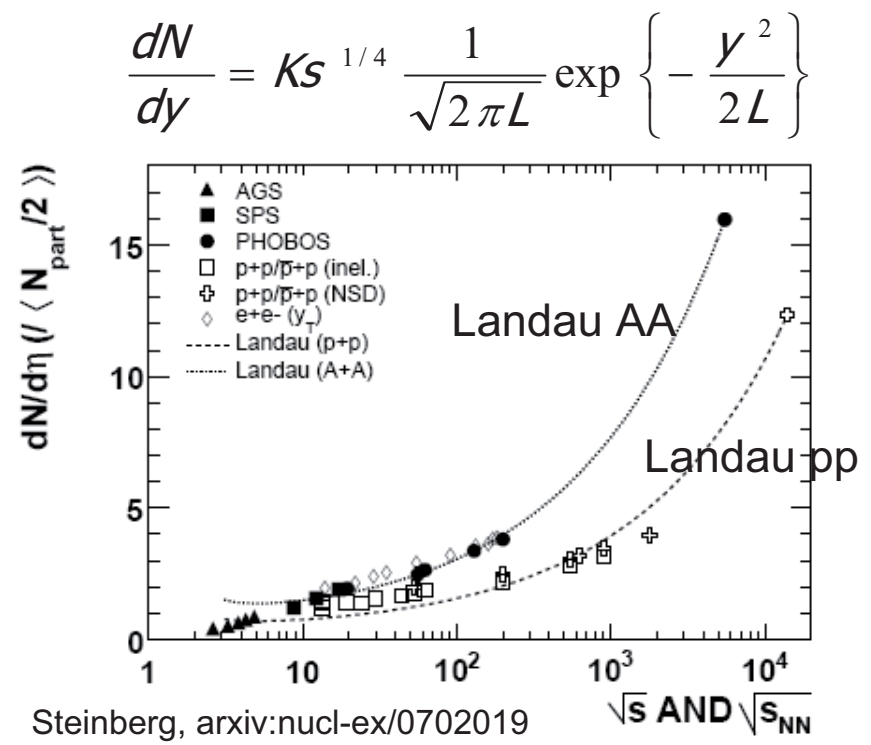

Landau hydrodynamics exhibits limiting fragmentation

$$
\begin{aligned}
& \frac{d N}{d y} \sim \frac{1}{\sqrt{L}} \exp \left\{-\frac{y^{\prime 2}}{2 L}-y^{\prime}\right\} \\
& y^{\prime}=y-y_{b} \quad \text { AA Collisions }
\end{aligned}
$$

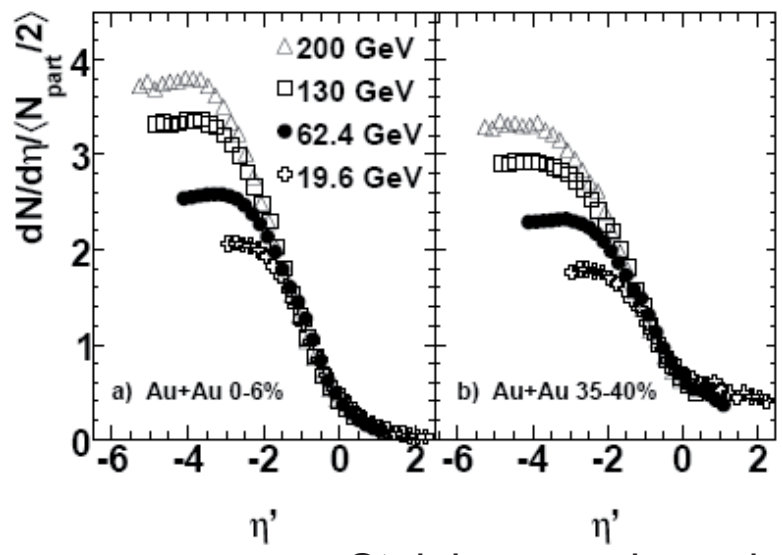

Steinberg, arxiv:nucl-ex/0702019 


\section{Unanwsered questions:}

- Is Landau's formula for y or $\eta$ ?

- Landau rapidity distribution is actually

$$
\frac{d N}{d y}=K s^{1 / 4} A_{\text {norm }} \exp \left\{\sqrt{L^{2}-y^{2}}\right\}
$$

The Gaussian Landau distribution is only an approximation

- Does the original Landau distribution agree with data?

We need to answer these questions in Landau hydrodynamics

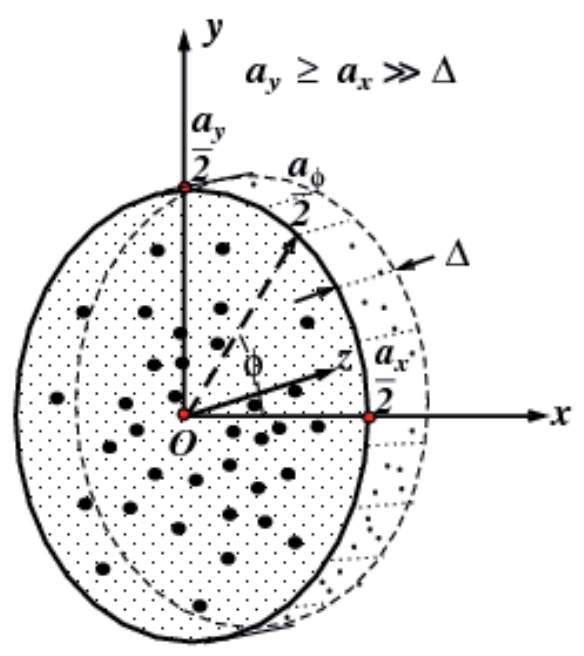

Initial configuration 


\section{Landau Hydrodynamics}

- Local thermal equilibrium

- Particle number is proportional to entropy

- Entropy is conserved in Landau hydrodynamics

- First stage, independent

(i) 1-D longitudinal expansion

(ii) transverse expansion

- Second stage of rapidity freeze-out when the transverse displacement is equal to the transverse dimension of the system

$$
\begin{gathered}
\mathrm{x}\left(\mathrm{t}_{\mathrm{m}}\right)=\mathrm{a} ; \quad \mathrm{t}_{\mathrm{m}}=\text { rapidity freeze-out time } \\
\frac{d N}{d y}=K \mathrm{~S}^{1 / 4} A_{\text {norm }} \exp \left\{\sqrt{L^{2}-y^{2}}\right\} \quad K \approx 2 .
\end{gathered}
$$

\section{Consrvation of Entropy in Landau hydrodynamics}

- Mean-free path is small, low viscosity

- The only means to destroy entropy conservation is shock wave.

- Landau hydrodynamics is applied after the completion of the compression stage. It deals only with the expansion of the dense matter after shock-wave compression 


\section{Landau hydrodynamics predictions on total $\mathrm{N}_{\underline{c h}}$}

$\otimes$ Hydrodynamical flow is isentropic (entropy conserving).

$\otimes$ Entropy $S$ is proportional to particle number $N$

$\otimes$ Conservation of entropy means that we can get

the total number of particles from the initial entropy.

Initial total energy $=E=\left(\sqrt{s_{N N}} / 2\right) 2 A=\sqrt{S_{N N}} A$

Initial volume $=V=\frac{4 \pi}{3} r_{0}^{3} A / \gamma ; \quad \gamma=\frac{\sqrt{s_{N N}}}{m_{p}}$

Energy density $=\varepsilon=\frac{E}{V}=\gamma \sqrt{s_{N N}} / \frac{4 \pi}{3} r_{0}^{3}$

Entr opy density $=\sigma=C \varepsilon^{3 / 4}=C\left(\gamma \sqrt{s_{N N}} / \frac{4 \pi}{3} r_{0}^{3}\right)^{3 / 4}$

Total entropy $=\sigma V=C\left(\gamma \sqrt{S_{N N}} / \frac{4 \pi}{3} r_{0}^{3}\right)^{3 / 4} \frac{4 \pi}{3} r_{0}^{3} A / \gamma$

$$
\propto\left(\sqrt{S_{N N}}\right)^{1 / 2} A
$$

Total number of particles $=N \propto\left(\sqrt{S_{N N}}\right)^{1 / 2} A$

$N_{c h} /\left(N_{\text {part }} / 2\right)=K\left(\sqrt{s_{N N}}\right)^{1 / 2} \quad K \approx 2$ for $\sqrt{s_{N N}}$ in $\mathrm{GeV}$.

\section{Landau prediction on $\mathrm{N}_{\mathrm{ch}} /\left(\mathrm{N}_{\text {part }} / 2\right)$ agrees with experiment}

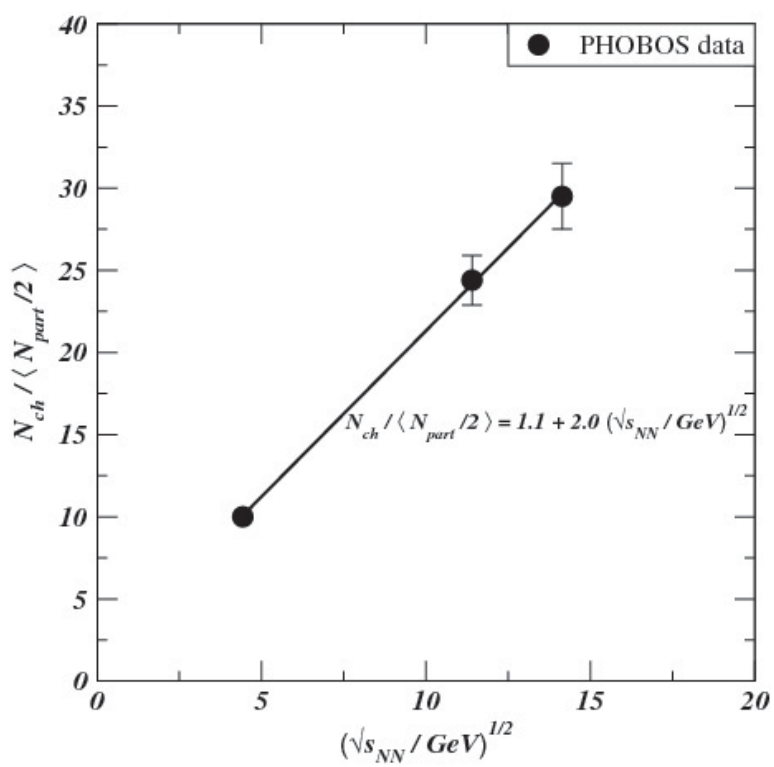




\section{1-D longitudinal expansion}

Hydrodynamic equations

$$
\begin{aligned}
& \frac{\partial T^{00}}{\partial t}+\frac{\partial T^{01}}{\partial Z}=0 \\
& \frac{\partial T^{01}}{\partial t}+\frac{\partial T^{11}}{\partial Z}=0 \\
& T^{\mu \nu}=(\varepsilon+p) u^{\mu} u^{v}-p g^{\mu \nu}
\end{aligned}
$$

We use simple equation of state

$p=\varepsilon / 3$

$$
\begin{array}{lll}
\text { Introduce } & u^{0}=\cosh y & u^{1}=\sinh y \\
& t_{+}=t+z \quad t_{-}=t-z
\end{array}
$$

Introduce logarithm of $t_{ \pm}$

$$
y_{ \pm}=\ln \left(t_{ \pm} / \Delta\right)
$$

\section{1-D longitudinal expansion solution}

Hydrodynamic equations becomes

$\frac{\partial \varepsilon}{\partial t_{-}}+2 \frac{\partial\left(\varepsilon e^{-2 y}\right)}{\partial t}=0$

$2 \frac{\partial\left(\varepsilon e^{+2 y}\right)}{\partial t_{+}}+\frac{\partial \varepsilon}{\partial t_{-}}=0$

Simple approximate solution

$\left\{\begin{array}{l}\varepsilon=\varepsilon_{0} \exp \left\{-\frac{4}{3}\left(y_{+}+y_{-}-\sqrt{y_{+} y_{-}}\right)\right\} \\ y=\left(y_{+}-y_{-}\right) / 2\end{array}\right.$

We can prove that this is an approximate solution by direct substitution.

Advantage: simplicity

Minor disadvanatges :

(i) only for the bulk part of fluid, not the edge part

(ii) limited choices of initial conditions 


\section{Solution of 1-D longitudinal expansion}

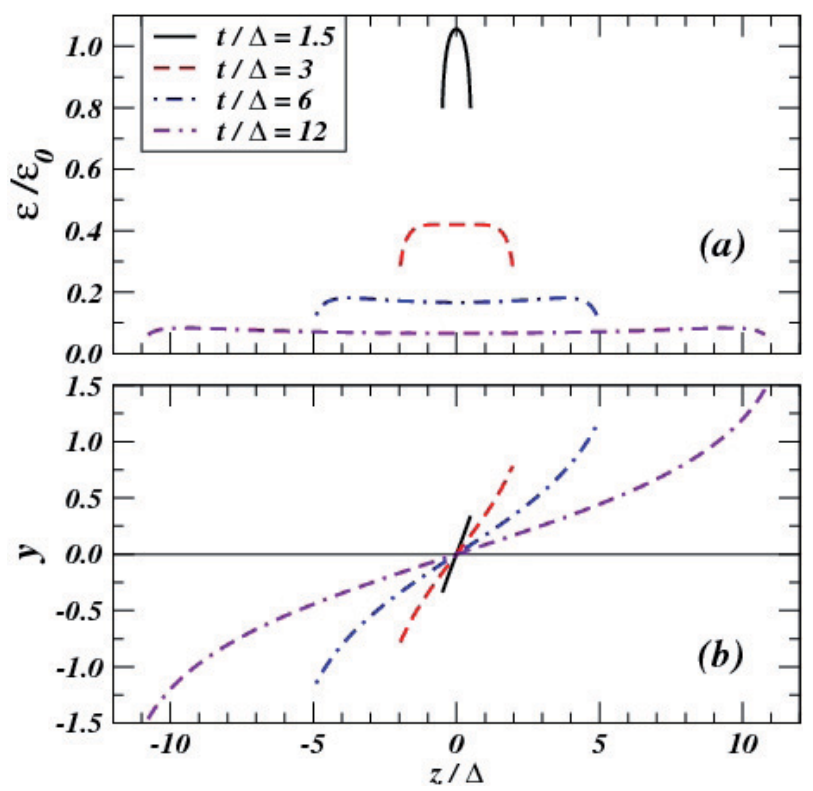

\section{Transverse expansion during the first stage}

Hydrodynamic equation $\quad \frac{\partial T^{02}}{\partial t}+\frac{\partial T^{02}}{\partial x}=0$

$T^{02}=(\varepsilon+p) u^{0} u^{2}=\frac{4}{3} u^{0} u^{0} v_{x} ; \quad u^{2}=u^{0} v_{x}$

$T^{22}=(\varepsilon+p) u^{2} u^{2}-p g^{22} \approx g$

Hydrodynamic equation becomes

$\frac{4}{3} \varepsilon u^{0} u^{0} \frac{\partial v_{x}}{\partial t}=-\frac{\partial p}{\partial x}=-\frac{1}{3} \frac{\partial \varepsilon}{\partial x}$

Transverse displacement is

$x(t)=\frac{1}{2} \frac{\partial v_{X}}{\partial t} t^{2} ; \quad \frac{\partial v_{X}}{\partial t}=\frac{2 x(t)}{t^{2}}$

Hydrodynamic equation is

$\frac{4}{3} \varepsilon u^{0} u^{0} \frac{2 x(t)}{t^{2}}=\frac{1}{3} \frac{\varepsilon}{a}$

$x(t)=\frac{t^{2}}{4 a u^{0} u^{0}}=\frac{t^{2}}{4 a \cosh ^{2} y}$

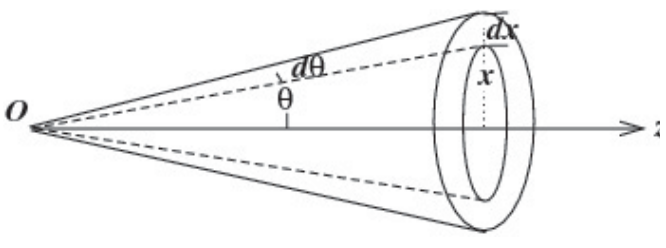




\section{Landau condition for rapidity freeze-out}

Angle (and rapidity ) will not change after the transverse displacement is equal to the transverse dimension.

$$
x(t)=\frac{t^{2}}{4 a \cosh ^{2} y}=a=\text { tranverse diameter }
$$

\section{Rapidity freeze-out occurs at different time $t$ for different rapidity (and z).}

\section{Rapidity distribution}

Rapidity distribution at time $t$

$$
\begin{aligned}
& d S=\sigma u^{0} d z \quad z=t \sinh y / \cosh y \\
& d S=\sigma u^{0} t d y / \cosh ^{2} y=\sigma t d y / \cosh y \\
& \sigma=c \varepsilon^{3 / 4} \\
& d S=c \varepsilon_{0}^{3 / 4} \exp \left\{-\left(y_{+}+y_{-}-\sqrt{y_{+} y_{-}}\right)\right\} t \frac{d y}{\cosh y} .
\end{aligned}
$$

We need the rapdity distribution at $t=t_{m}(y)$

$$
\begin{aligned}
& d S=C \varepsilon_{0}^{3 / 4}\left[\exp \left\{-\left(y_{+}+y_{-}-\sqrt{y_{+} y_{-}}\right)\right\} t\right]_{\left(t=t_{m}(y)\right)} \frac{d y}{\cosh y} \\
& y_{ \pm}=\ln \left(\frac{2 a}{\Delta}\right) \pm y=y_{b} \pm y
\end{aligned}
$$

Final entropy distribution

$d S \propto \exp \left\{\left(2 y_{b}-\sqrt{y_{b}^{2}-y^{2}}\right)\right\} d y$

$N \propto S$

Final rapidity distribution : $\frac{d N}{d y} \propto \exp \left\{\sqrt{y_{b}^{2}-y^{2}}\right\}$ 


\section{Comparison with experiment}

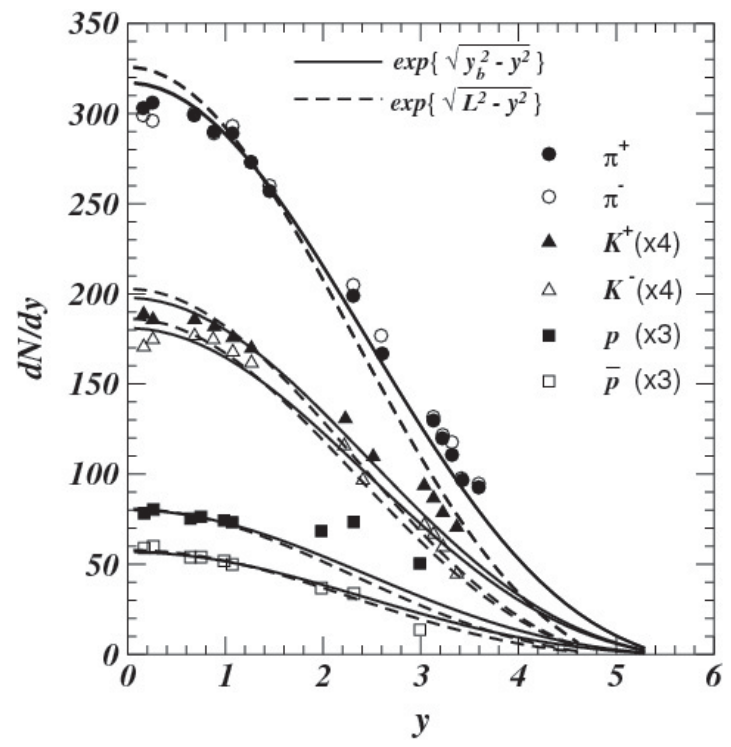

Modified distribution gives better agreement than Landau distribution

\section{Comparison with experiment}

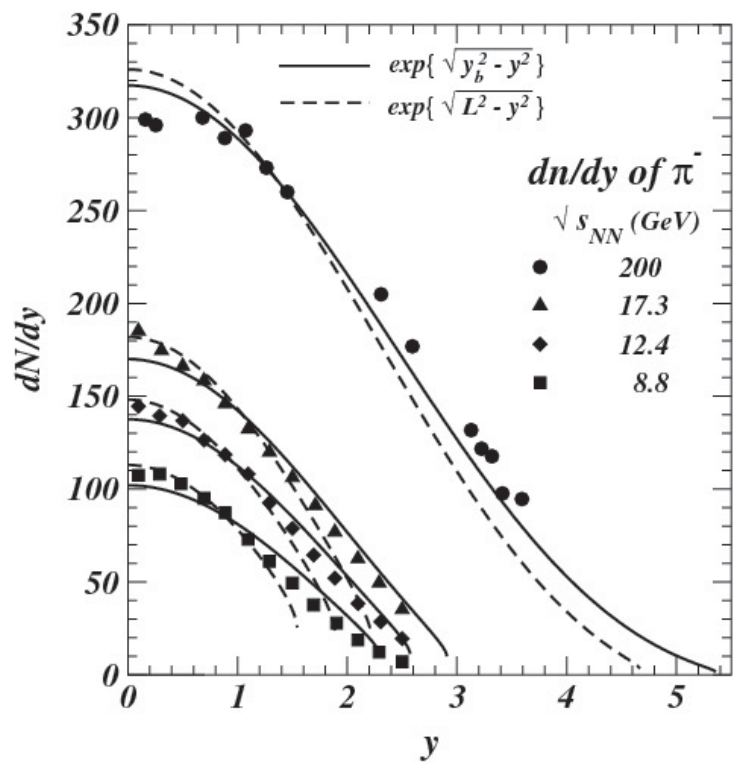

Modified distribution gives better agreement than Landau distribution 


\section{Conclusions}

- Landau's prediction on $\mathrm{N}_{\mathrm{ch}}$ agrees with data

- The rapidity distribution in Landau hydrodynamics should be modified. The modified rapidity distribution gives better agreement with experimental data than the Landau distribution

- The quantitative agreement of Landau hydrodynamics supports its use in other problems of heavy ion collisions, such as J/psi suppression, jet quenching, and ridge jetmedium interaction,....

\section{Quarkonia in Quark-Gluon Plasma}

Cheuk-Yin Wong

\section{Oak Ridge National Laboratory}

- Introduction

- Potential model of quarkonia in QGP using lattice gauge thermodynamic quantities

- Stability of quarkonia in QGP

- Effect of dynamical quarks on quarkonia stability

- Quark drip lines for quarkonia with light quarks

- Conclusions

C.Y.Wong,E.S.Swanson,T.Barnes,Phys.Rev.C65,014903('02)

C.Y.Wong,Phys.Rev.C65,034902('02)

C.Y.Wong,J.Phys.G28,2349('O2)

W.N.Zhang,C.Y.Wong,Phys.Rev.C68,035211('03)

C.Y.Wong,Phys.Rev.C72,034906('05)

C.Y.Wong,J.Phys.G32,S301('06)

C.Y.Wong,Phys.Rev.C76:014902('07)

C.Y.Wong,H.Crater,Phys.Rev.D75,034505('07) 


\section{Why study quarkonia in quark-gluon plasma?}

- Heavy quarkonia may be used as a plasma diagnostic tool

- Successes of the recombination model

- Successes of the thermal mode

--- quarkonia with light quarks may be stable in quark-gluon plsama, at least near Tc

\section{Q-Qbar interaction in Quarkonia}

- The screening between Q and Qbar in QGP is non-perturbative in nature and must be studied non-perturbatively.

- Need lattice gauge theory

- Need to understand the results from lattice gauge theory

- Need to study dynamical effects in screening, response and relaxation of the medium to Q-Qbar motion 


\section{What kind of framework in lattice gauge theory?}

- Lattice is a subsystem in a much large thermal bath

- Subsystem is in constant contact with bath

- In the grand-canonical ensemble (which is used in the lattices gauge calculations), the subsystem particle numbers and energy of the subsystem are adjusted to maintain the same temperature as the bath

\section{Types of lattice calcualtions}

- Polyakov lines $\left\langle\operatorname{tr}\left(L(r / 2) L^{+}(-r / 2)\right\rangle\right.$

- Meson correlators

$$
\left\langle\psi_{1}\left(x_{1}\right) O \psi_{2}\left(x_{2}\right) \psi_{1}^{+}\left(x_{1}{ }^{\prime}\right) O \psi_{2}{ }^{+}\left(x_{2}{ }^{\prime}\right)\right\rangle
$$

Gauge invariant correlator

$$
\left\langle\psi_{1}(\overrightarrow{0}, t) O \psi_{2}(\overrightarrow{0}, t) \psi_{1}^{+}(\overrightarrow{0}, 0) O \psi_{2}^{+}(\overrightarrow{0}, 0)\right\rangle
$$




\section{Why study heavy quarkonia in a hot medium?}

Two new surprising results from lattice gauge calculations

- Lattice spectral function analyses in quenched QCD show that $\mathrm{J} / \psi$ is stable up to $1.6 \mathrm{Tc}$

- Lattice static Q-Q "potential” appears to be very strong between 1 and 2 Tc

- Shuryak, Zahed, Brown, Lee, and Rho suggested that even light quarkonia may be bound in quark-gluon plasma

$\square$ We need

- to confirm these lattice gauge results

- to study effects of dynamical quarks on $J / \psi$ stability

- $\quad$ to assess the strength of the Q-Q potential

- $\quad$ to examine the stability of quarkonia

Lattice gauge spectral analyses in the quenched approximation show that the width of $\mathrm{J} / \Psi$ remains narrow up to $\mathrm{T} \leq 1.6 \mathrm{~T}_{\mathrm{C}}$

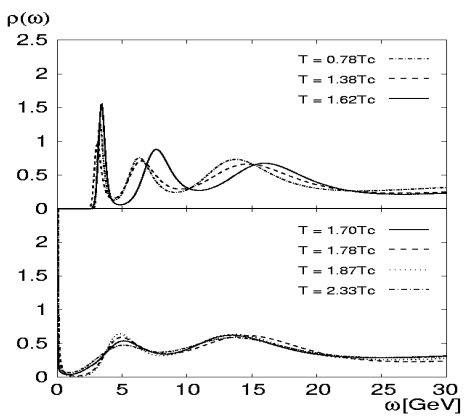

M. Asakawa, T. Hatsuda, and Y. Nakahara, Nucl. Phys. A715, 863 (03)

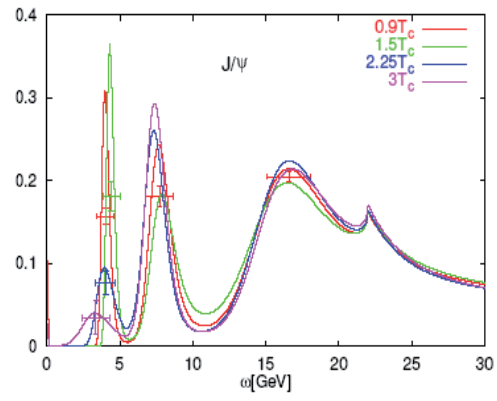

S. Datta, F. Karsch, P. Petreczky, and I. Wetzorke, Phys. Rev. D69,094507(04)

The drastic change of the spectral function between 1.62-1.70Tc suggests the occurrence of spontaneous dissociation at 1.62-1.70Tc. 


\section{Why mass of $\mathrm{J} /$ psi roughly constant of $\mathrm{T}$ ?}

- In Debye screening, the screening potential is

$V(r)=-\frac{\alpha \exp \{-\mu r\}}{r}-\frac{\alpha}{r_{D}}=-\frac{\alpha \exp \{-\mu r\}}{r}-\alpha \mu$

The mass of the system is constant to first order in $\mu$

\section{Questions:}

1 What does the potential model say about the $\mathrm{J} / \psi$ spontaneous dissociation temperature?

2 What are the effects of dynamical quarks?

3 What is the strength of interaction between a static quark and antiquark? 
Kaczmarek et al. calculated the color-singlet $\mathrm{F}_{1}$ and $\mathrm{U}_{1}$ in the quenched approximation [hep-lat/0309121]

$$
\begin{aligned}
& \left\langle\operatorname{TrL}(\mathrm{r}) \mathrm{L}^{+}(0)\right\rangle=\mathrm{e}^{-\mathrm{F}_{1}(\mathrm{r}, \mathrm{T}) / \mathrm{T}} \\
& \mathrm{U}_{1}(\mathrm{r}, \mathrm{T})=\mathrm{F}_{1}(\mathrm{r}, \mathrm{T})-\mathrm{T} \frac{\partial \mathrm{F}_{1}(\mathrm{r}, \mathrm{T})}{\partial \mathrm{T}} \\
& \mathrm{U}_{1}(\mathrm{r}, \mathrm{T})=\mathrm{F}_{1}(\mathrm{r}, \mathrm{T})+\mathrm{T} \mathrm{S}_{1}(\mathrm{r}, \mathrm{T})
\end{aligned}
$$

$F_{1}(r, T)$ was calculated in the Coulomb gauge

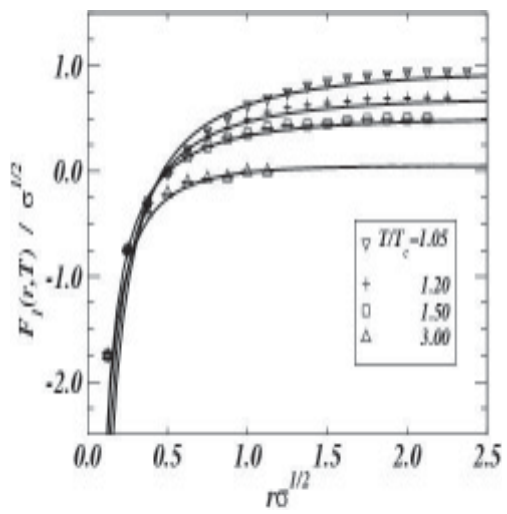

$U_{1}$ is much deeper than $F_{1}$ and can hold many more bound states

\section{What is the Q- $\bar{Q}$ potential?}

\section{Initial conjectures:}

1. $\mathrm{F}_{1}(\mathrm{r}, \mathrm{T})$ is the $\mathrm{Q}-\overline{\mathrm{Q}}$ potential

(Digal et.al `01,Wong `02,Blaschke et.al 05)

2. $\mathrm{U}_{1}(\mathrm{r}, \mathrm{T})$ is the $\mathrm{Q}-\overline{\mathrm{Q}}$ potential

$$
\mathrm{U}_{1}(\mathrm{r}, \mathrm{T})=\mathrm{F}_{1}(\mathrm{r}, \mathrm{T})+\mathrm{TS}_{1}(\mathrm{r}, \mathrm{T})
$$

(Kaczmarek et al.`02, Shuryak et al `04) 


\section{To extract $\mathrm{Q}-\overline{\mathrm{Q}}$ potential from lattice gauge $\mathrm{F}_{1}$ and $\mathrm{U}_{1}$ ?}

- We need to understand the meaning of $U_{1}$. - We need to understand the meaning of $\mathrm{TS}_{1}=\mathrm{U}_{1}-\mathrm{F}_{1}$.

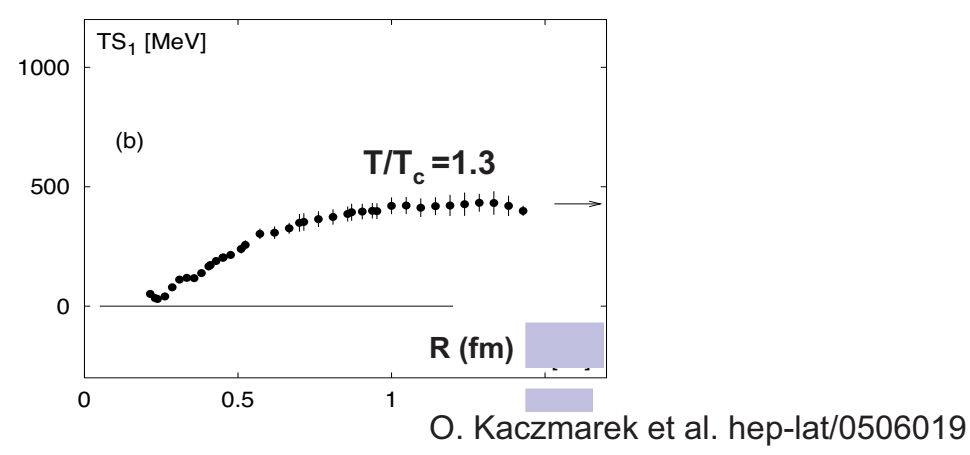

Why such behavior?

Thermodynamical Quantities $\mathrm{U}_{1}, \mathrm{~S}_{1}, \mathrm{~F}_{1}$

- Internal energy $\mathrm{U}_{1}$ (kinetic plus potential energies)

- Entropy $S_{1}$ (the degree of different ordering)

$$
S_{1}=-\sum\left\{n_{i} \ln n_{i}-\left(1+n_{i}\right) \ln \left(1+n_{i}\right)\right\}
$$

$\mathrm{TS}_{1}$ is the energy associated with entropy

- Free energy $\mathrm{F}_{1}=\mathrm{U}_{1}-\mathrm{TS}_{1}$

( the internal energy minus the energy associated with entropy) 


\section{Thermodynamical Quantities}

- Internal energy U (kinetic plus potential energies)

- Entropy S (the degree of different ordering)

$$
S=\sum\left\{n_{i} \ln n_{i}-\left(1+n_{i}\right) \ln \left(1+n_{i}\right)\right\}
$$

TS is the energy associated with entropy

- Free energy $\mathrm{F}=\mathrm{U}$ - TS

( the internal energy minus the energy associated with entropy)

\section{Equilibrium condition}

- $\mathrm{dU}=\mathrm{T} \mathrm{dS}-\mathrm{P} \mathrm{dV}$

Define free energy $\mathrm{F}=\mathrm{U}-\mathrm{TS}$, we get $\mathrm{dF}=-\mathrm{SdT}-\mathrm{PdV}$

If the temperature is held fixed (thermal bath) and the volume does not change locally, then $\mathrm{F}$ is a constant.

- In a constant temperature environment, equilibrium is reached when $\mathrm{dF}=0$,

i.e. the variation of $\mathrm{F}$ with respect to all degrees of freedom is zero in a thermal bath. 


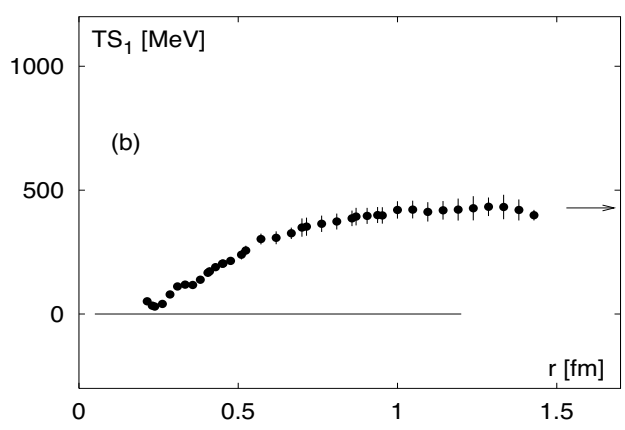

Entropy increases as $Q$ and Qbar move away from each other

The number of gluons increases as $Q$ and Qbar move away from each other because the first order of screening cancels but the second order change of gluon number always increases.

We want Q-Qbar interaction without changing the gluon internal energy.

We therefore need to subtract out the increases in gluon energy when $Q$ and $Q b a r$ changes separations.

We find in this procedure that the $\mathrm{Q}-\overline{\mathrm{Q}}$ potential is

$$
\boldsymbol{U}_{Q \bar{Q}}^{(1)}=\boldsymbol{U}_{1}-\boldsymbol{U}_{g}
$$

By the first law of thermodynamics,

$$
\begin{aligned}
& \frac{d U_{g}}{d V}=\varepsilon=T \frac{d S_{g}}{d V}-p \quad\left(S_{g} \text { is the gluon entropy }\right) \\
& p=\frac{3 p}{\varepsilon} \frac{d U_{g}}{3 d V}=a(T) \frac{d U_{g}}{3 d V} \\
& a(T)=3 p / \varepsilon \text { is known from the equation of state }
\end{aligned}
$$

So, $\quad \frac{d U_{g}}{d V}=\frac{3}{3+a} T \frac{d S_{g}}{d V}$,

and $\quad \boldsymbol{U}_{g}=\frac{\mathbf{3}}{\mathbf{3}+\boldsymbol{a}} \boldsymbol{T} \boldsymbol{S}_{g} . \quad$ But $\quad \boldsymbol{T} \boldsymbol{S}_{g}=\boldsymbol{U}_{\mathbf{1}}-\boldsymbol{F}_{\mathbf{1}}$

Hence, $U_{Q \bar{Q}}^{(1)}=U_{1}-U_{g}=U_{1}-\frac{3}{3+a}\left(U_{1}-F_{1}\right)$

$$
U_{Q \bar{Q}}^{(1)}=\frac{3}{3+a} F_{1}+\frac{a}{3+a} U_{1}
$$




\section{$F_{1}$ and $U_{1}$ fractions depend on $T$}

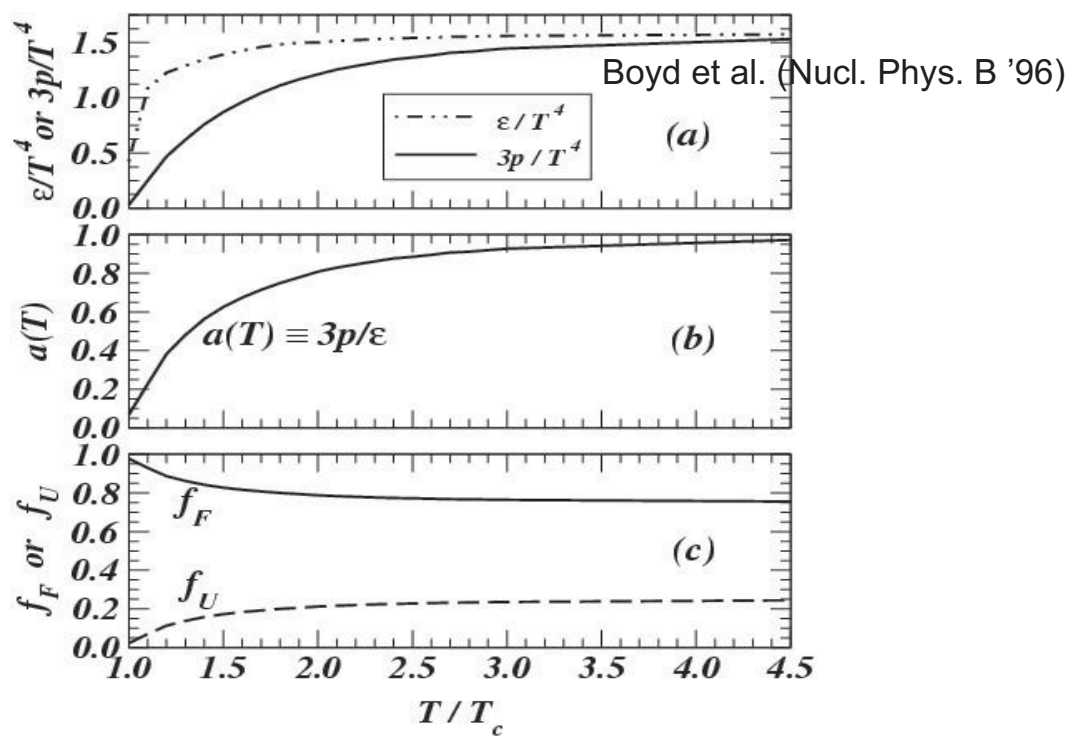

\section{Solve for $\mathrm{Q}-\overline{\mathrm{Q}}$ bound states}

$$
\left\{-\frac{\nabla^{2}}{2 \mu_{\text {red }}}+\left[\begin{array}{c}
U_{Q \bar{Q}}^{(1)}(r, T)-U_{Q \bar{Q}}^{(1)}(r \rightarrow \infty, T) \\
F_{1}(r, T)-F_{1}(r \rightarrow \infty, T) \\
U_{1}(r, T)-U_{1}(r \rightarrow \infty, T)
\end{array}\right]\right\} \psi(r, T)=\varepsilon(T) \psi(r, T)
$$

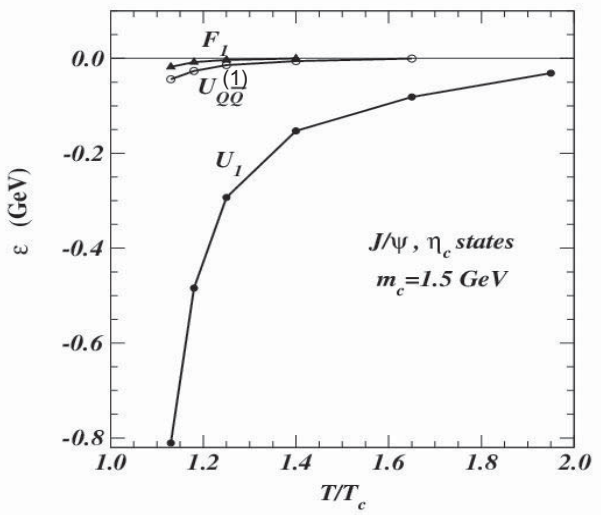




\section{Spontaneous dissociation temperatures in}

\section{quenched QCD}

\begin{tabular}{|c|l|l|l|l|}
\hline $\begin{array}{l}\text { Heavy } \\
\text { Quarkonium }\end{array}$ & $\begin{array}{l}\text { Spectral } \\
\text { Analysis }\end{array}$ & $\begin{array}{c}U_{Q \bar{\sigma}}^{(1)} \\
\text { Potential }\end{array}$ & $\begin{array}{l}\mathrm{F}_{1} \\
\text { Potential }\end{array}$ & $\begin{array}{l}\mathrm{U}_{1} \\
\text { Potential }\end{array}$ \\
\hline $\mathrm{J} / \Psi$ & $1.62-1.70 \mathrm{~T}_{\mathrm{C}}$ & $1.62 \mathrm{~T}_{\mathrm{C}}$ & $1.40 \mathrm{~T}_{\mathrm{C}}$ & $2.60 \mathrm{~T}_{\mathrm{C}}$ \\
\hline $\mathrm{X}_{\mathrm{c}}, \Psi^{\prime}$ & below $1.1 \mathrm{~T}_{\mathrm{C}}$ & unbound & unbound & $1.18 \mathrm{~T}_{\mathrm{C}}$ \\
\hline $\mathrm{Y}$ & & $4.10 \mathrm{~T}_{\mathrm{C}}$ & $3.50 \mathrm{~T}_{\mathrm{C}}$ & $\sim 5.0 \mathrm{~T}_{\mathrm{C}}$ \\
\hline$\chi_{\mathrm{b}}$ & $1.15-1.54 \mathrm{~T}$ & $1.19 \mathrm{~T}$ & $1.10 \mathrm{~T}_{\mathrm{C}}$ & $1.73 \mathrm{~T}_{\mathrm{C}}$ \\
\hline
\end{tabular}

The comparison of the spontaneou s dissociation temperatures in quenched QCD indicates that the $\boldsymbol{U}_{\boldsymbol{Q} \bar{Q}}^{(\mathbf{1})}$ potential,

$$
U_{Q \bar{Q}}^{(1)}=\frac{3}{3+a} F_{1}+\frac{a}{3+a} U_{1},
$$

may be the appropriate $\boldsymbol{Q} \overline{\boldsymbol{Q}}$ potential to study quarkonium stability. We may also use the $U_{Q \bar{Q}}^{(1)}$ potential in full QCD to study quarkonium stability. 


\section{Quenched QCD \& Full QCD}

- Quenched QCD is inadequate as it neglects the effects of dynamical quarks

- We need to study full QCD with dynamical quarks

- Kaczmarek et al. (PRD 71, 114510 '05) have obtained $\mathrm{F}_{1}$ and $U_{1}$ in full QCD (with 2 flavors)

- Karsch et al. has obtained a(T) (PLB 478, 447).

- We can use the potential model to study the stability of quarkonium in full QCD

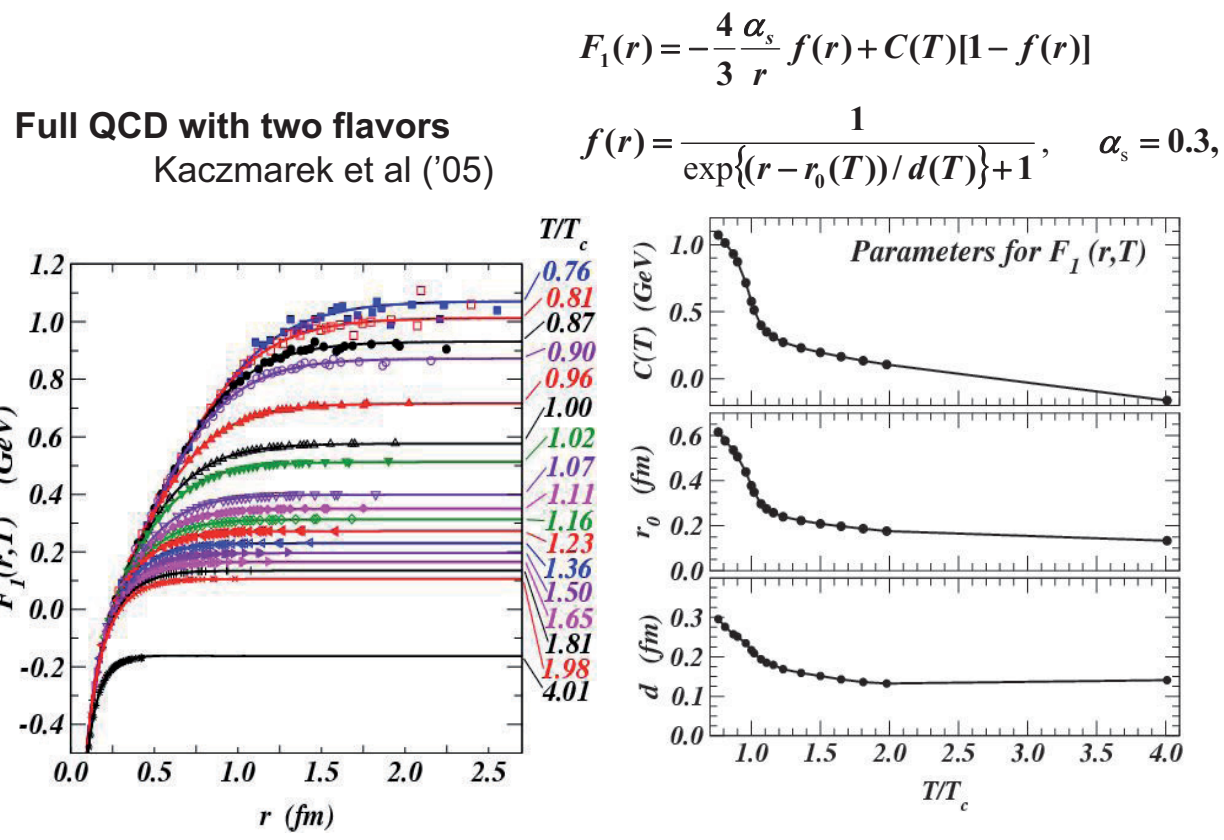


$\begin{array}{ll}\text { Full QCD with two flavors } \quad f(r) & =\frac{4}{\exp \left\{\left(r-r_{0}(T)\right) / d(T)\right\}+1}, \quad \alpha_{\mathrm{s}}=0.3,\end{array}$
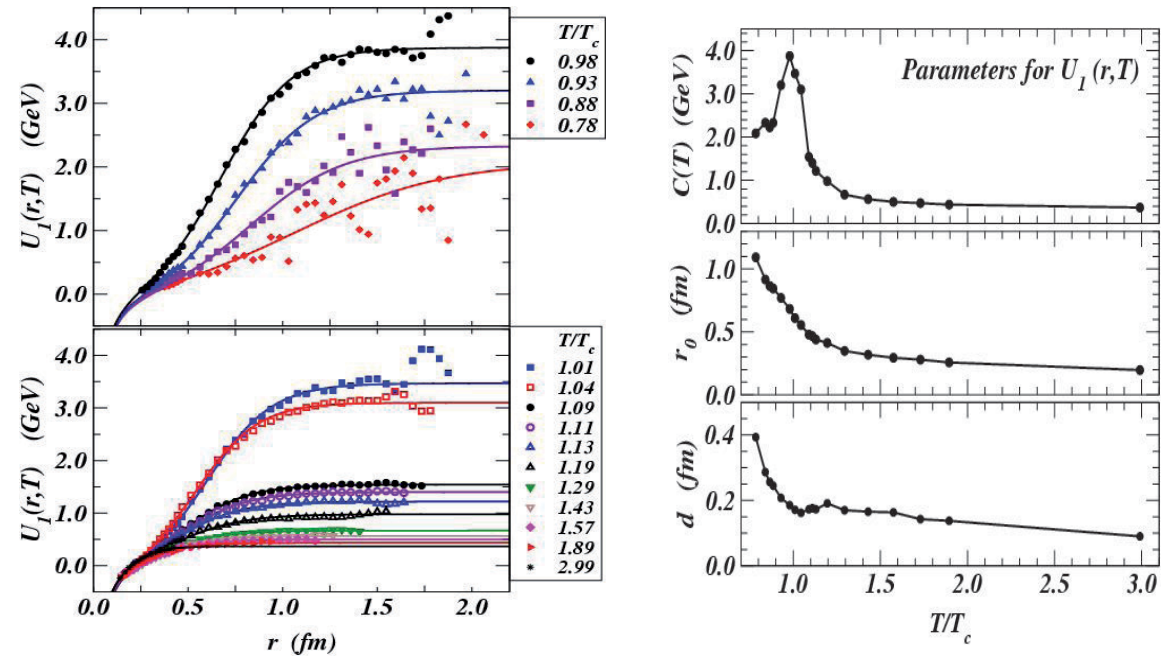

Spontaneous dissociation temperatures in quenched QCD \& full QCD

\begin{tabular}{|c|l|l|l|}
\hline $\begin{array}{c}\text { Heavy } \\
\text { Quarkonium }\end{array}$ & $\begin{array}{l}\text { Quenched } \\
\text { QCD } U_{Q \bar{Q}}^{(1)}\end{array}$ & $\begin{array}{l}\text { 2-flavor } \\
\text { QCD } U_{Q \bar{Q}}^{(1)}\end{array}$ & $\begin{array}{l}\text { Spectral } \\
\text { Analysis } \\
\text { Quenched } \\
\text { QCD }\end{array}$ \\
\hline $\mathrm{J} / \Psi$ & $1.62 \mathrm{~T}_{\mathrm{C}}$ & $1.42 \mathrm{~T}_{\mathrm{C}}$ & $\sim 1.6 \mathrm{~T}_{\mathrm{C}}$ \\
\hline $\mathrm{X}_{\mathrm{c}}, \Psi^{\prime}$ & unbound & unbound & below $1.1 \mathrm{~T}_{\mathrm{C}}$ \\
\hline $\mathrm{Y}$ & $4.10 \mathrm{~T}_{\mathrm{C}}$ & $3.30 \mathrm{~T}_{\mathrm{C}}$ & \\
\hline$\chi_{\mathrm{b}}$ & $1.18 \mathrm{~T}_{\mathrm{C}}$ & $1.22 \mathrm{~T}_{\mathrm{C}}$ & $1.15-1.54$ \\
\hline
\end{tabular}


Recent lattice calculations in

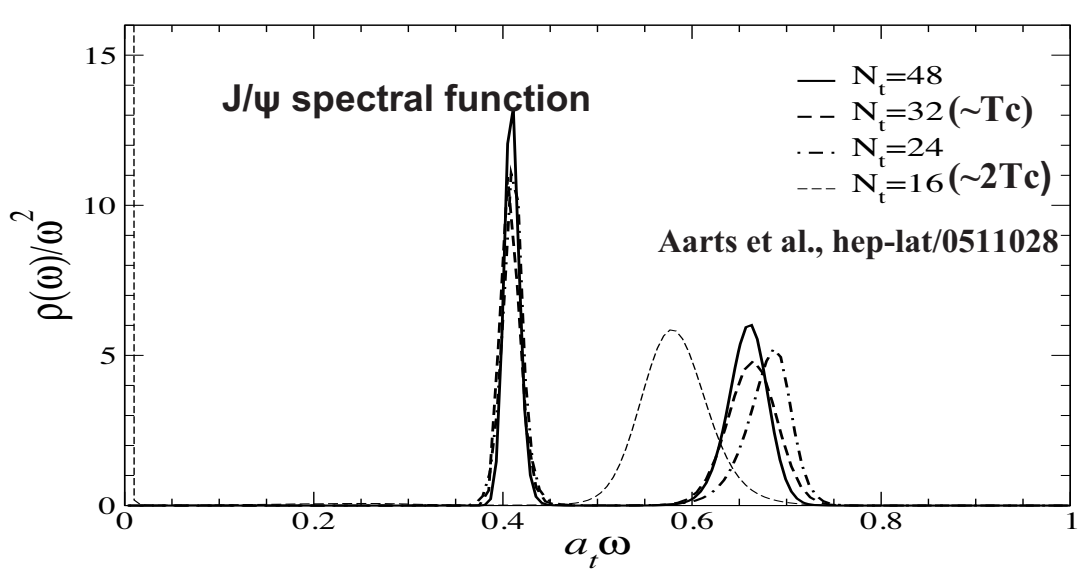

$\mathrm{J} / \Psi$ appears to have narrow width even up to $\sim 2 \mathrm{Tc}$ in full QCD

\section{Quarkonia in quark-gluon plasma}

The Q-Qbar potential extracted from lattice calculations can be used to examine the stability of quarkonia of different quark masses.

We can treat the quark mass as a variable and obtain the spontaneous dissociation temperature as a function of the reduced mass. 


\section{The quark drip line}

- The quark drip line is the line in the $(\mu, \mathrm{T})$ space above which Q-Qbar is unbound.

- It can also be characterized by the nature of the Q-Qbar state: 1s drip line, 1p drip line,..

- Given the Q-Qbar potential, the drip line can be determined by locating the spontaneous dissociation temperature as a function of the reduced mass.

\section{Quark drip lines in quark-gluon} plasma in quenched QCD

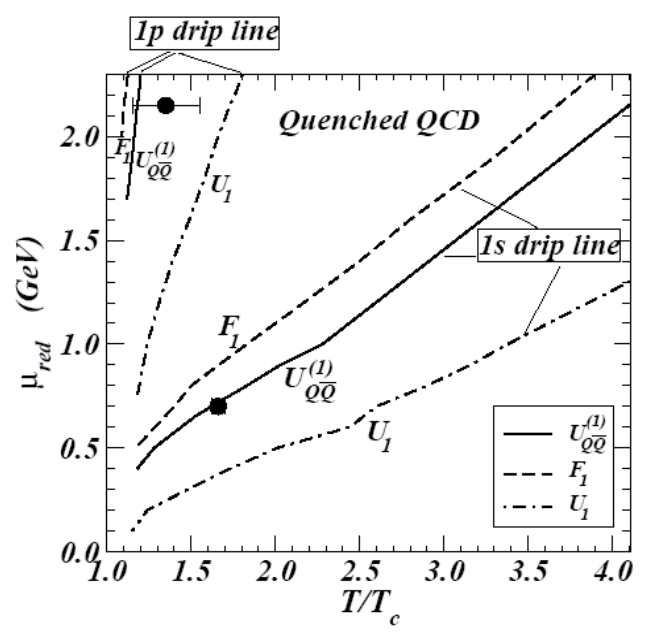




\section{Stability of quarkonia in quark-gluon plasma in full QCD}

Dynamical quarks modifies the 1s drip line but not the $1 \mathrm{p}$ drip line.

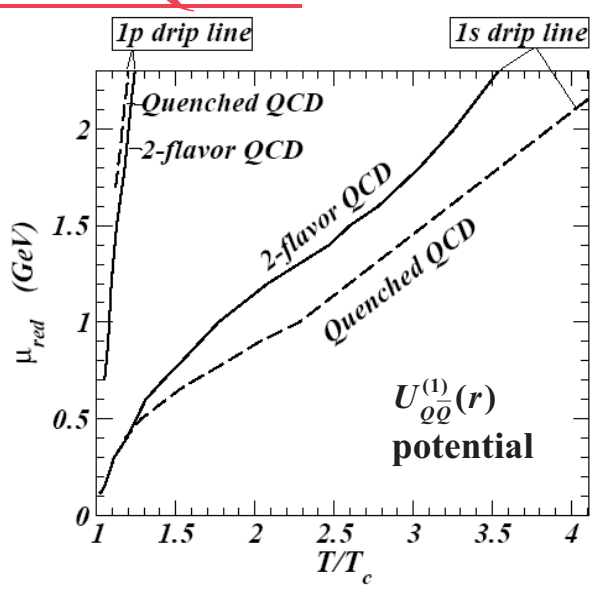

\section{Can the potential model be applied to light quarkonia?}

- Because of the strong coupling, light quarks becomes quasiparticles and acquire masses

- The quasiparticle masses of quarks in quark-gluon plasma can be estimated by looking at the equation of state (Levai et al., Szabo et al, Iavanov et al.). They found that the quasi-particle masses of $\mathrm{u}, \mathrm{d}$, and $\mathrm{s}$ quarks are 0.3-0.4 GeV for $\mathrm{Tc}<\mathrm{T}<2 \mathrm{Tc}$. 


\section{$\underline{\text { Light quark quarkonia }}$}

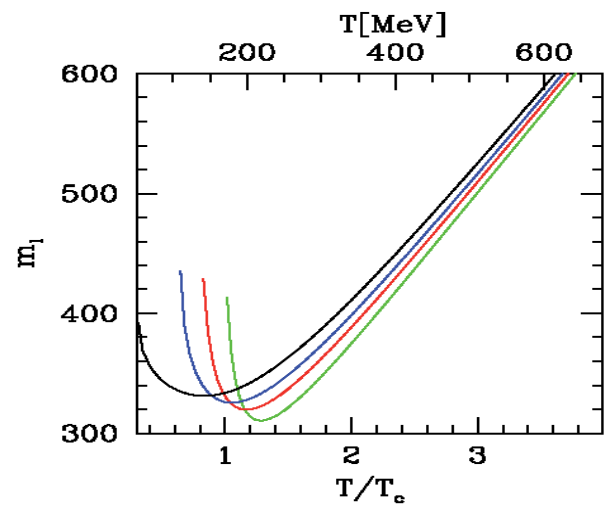

For light quarks with a mass of 300-400 MeV, quarkonia with light quarks can be stable up to $1.06 \mathrm{Tc}$.

Szabo et al. JHEP 0306, 008 ( 03 )

Results from Levai et al and Ivanov et al are similar.

\section{Conclusions}

- The potential model is consistent with the lattice gauge spectral function analysis, if the Q-Qbar potential is a linear combination of $\mathrm{F}_{1}$ and $\mathrm{U}_{1}$, with coefficients depending on the equation of state.

- The effects of the dynamical quarks modifies only slightly the stability of $\mathrm{J} / \psi$. $\mathrm{J} / \psi$ dissociates spontaneously at about $1.62 \mathrm{Tc}$ in quenched QCD and at $1.42 \mathrm{Tc}$ in 2flavor QCD.

- The interaction between a static quark and antiquark is such that the quark drip lines limit possible quarkonium states with light quarks to $\mathrm{T}$ close to Tc. 


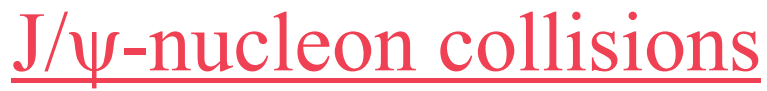

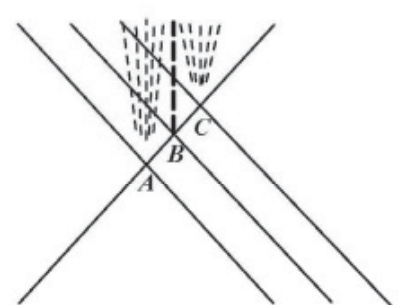

(a)

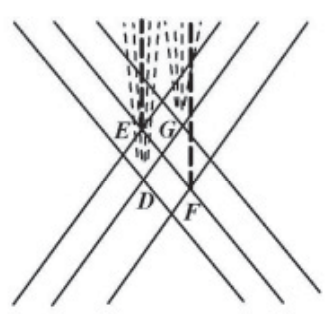

(b)

- $\mathrm{J} / \psi$ precursor collide with nucleons at high energy

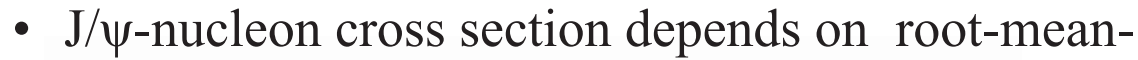
square separation

- $\mathrm{J} / \psi$ colides with comovers at low energy

\section{J/psi-nucleon high energy cross section}

Zhang \& Wong, PRC68,035211(03)

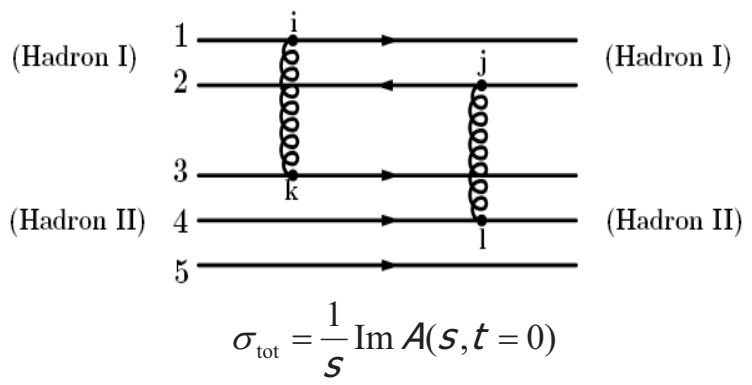

$$
\begin{gathered}
A(s, t)=\sum_{i j k \ell} \int d \vec{b} e^{-i \vec{Q} \cdot \vec{b}}\left\langle\Psi_{I}(i j) \Psi_{I I}(k /)\left|V_{T}(i k) V_{T}(j /)\right| \Psi_{I}(i j) \Psi_{I I}(k /)\right\rangle \\
V_{T}(\vec{b})=\int_{-\infty}^{\infty} d z V(\vec{r}) \\
Q^{2}=-t
\end{gathered}
$$


EPJ Web of Conferences

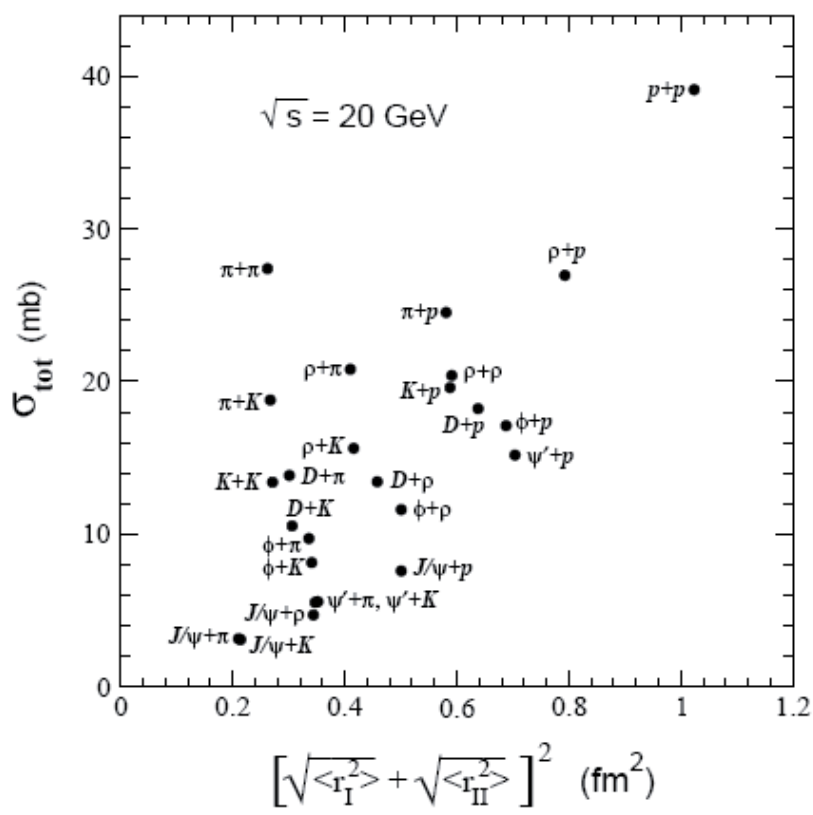

Zhang \& Wong, PRC68,035211(03)

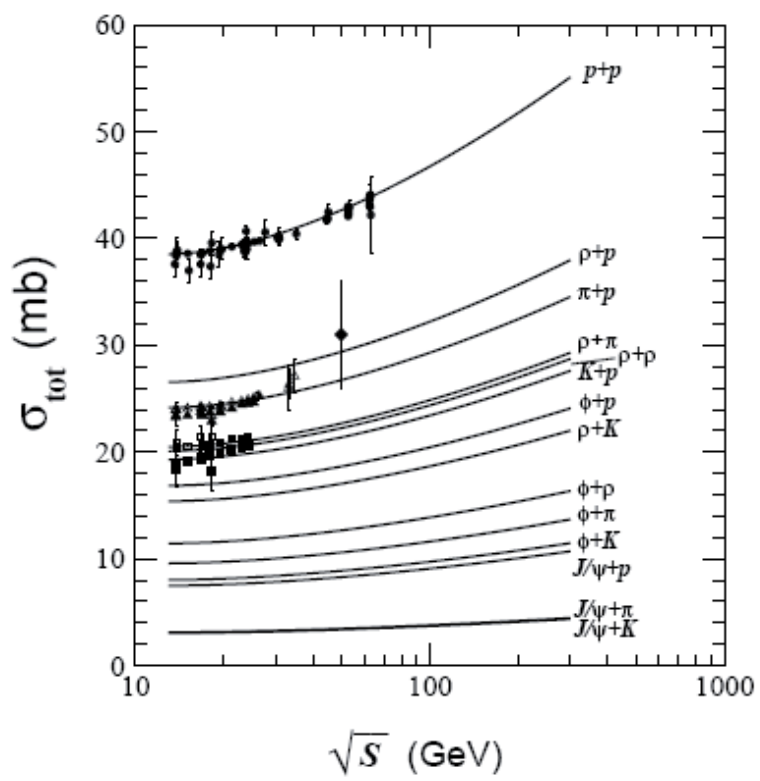

01006-p.46 


\section{Cross section dependence on color-octet admixture \& c-cbar separation}

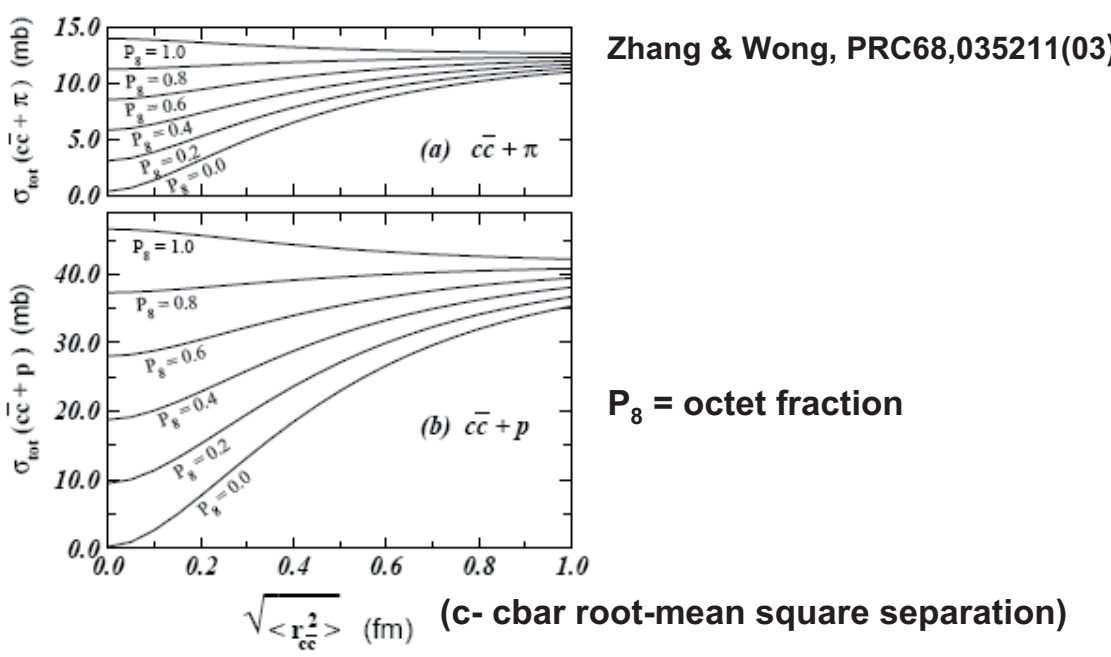

For color-singlet $\mathrm{C}$-Cbar, cross section increases with $\mathrm{C}$-Cbar separation.

\section{Low-energy $\pi$-J/ $\psi$ cross section}

\section{Use the Barnes-Swanson quark-interchange model}

Martins,Blaschke,Quack, PRC 51, 2723 ('95)

C.Y.Wong, E. Swanson, T. Branes, PRC65,014903('010

$$
\frac{d \sigma}{d t}=\frac{1}{64 \pi s\left|\vec{p}_{A}\right|^{2}}\left|M_{f i}\right|^{2}
$$

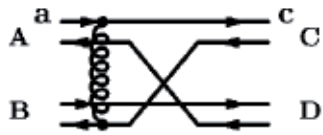

C1

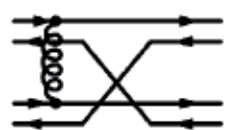

$\mathrm{T} 1$

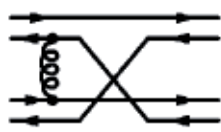

$\mathrm{C} 2$

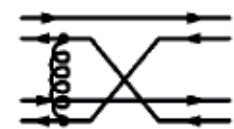

$\mathrm{T} 2$ 
EPJ Web of Conferences

\section{Test the formulation in $\pi-\pi$ scattering}

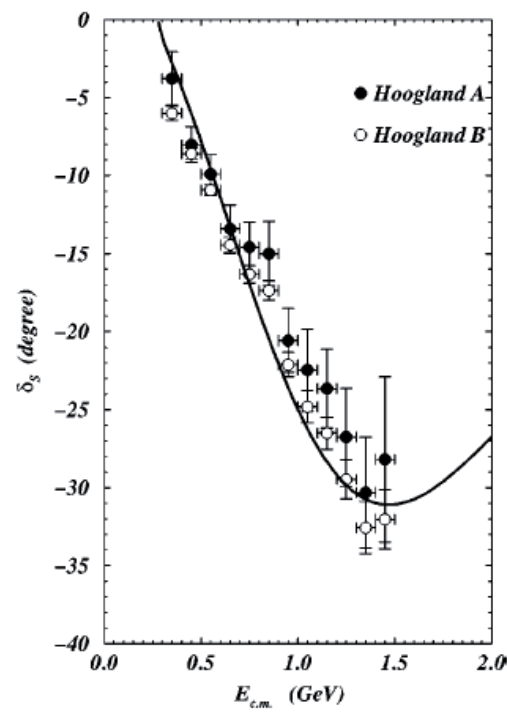

FIG. 4. Comparison of our theoretical $I=2 \pi \pi$ phase shift (solid curve) with the data of Hoogland et al. [41].
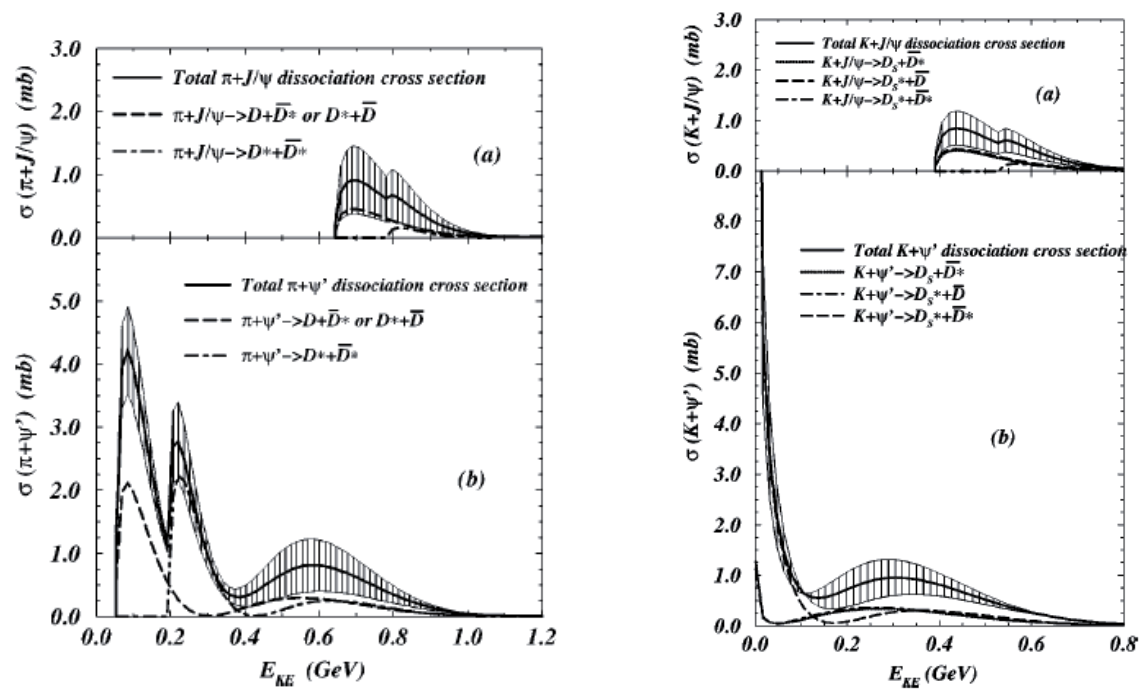

Wong, Swanson, Barnes PRC 65014903 (2001) 


\section{$\mathrm{J} / \Psi$ dissociation by collision with gluon}

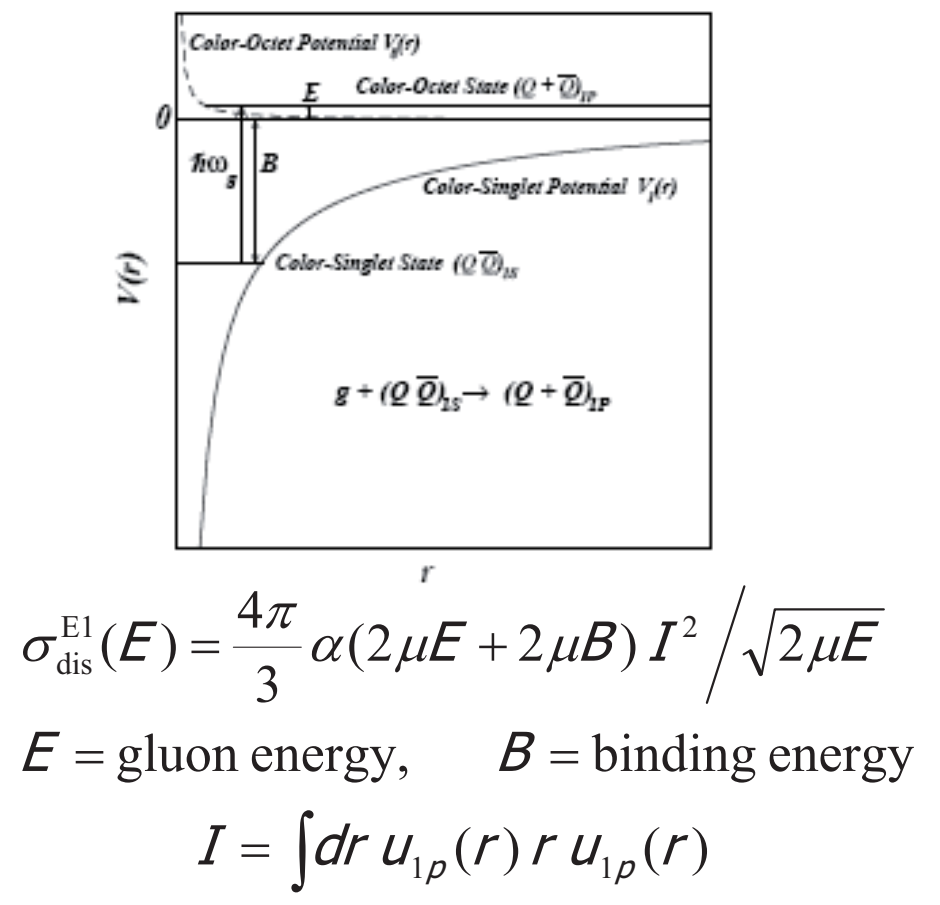

\section{$\mathrm{J} / \Psi$ dissociation in collision with gluons}

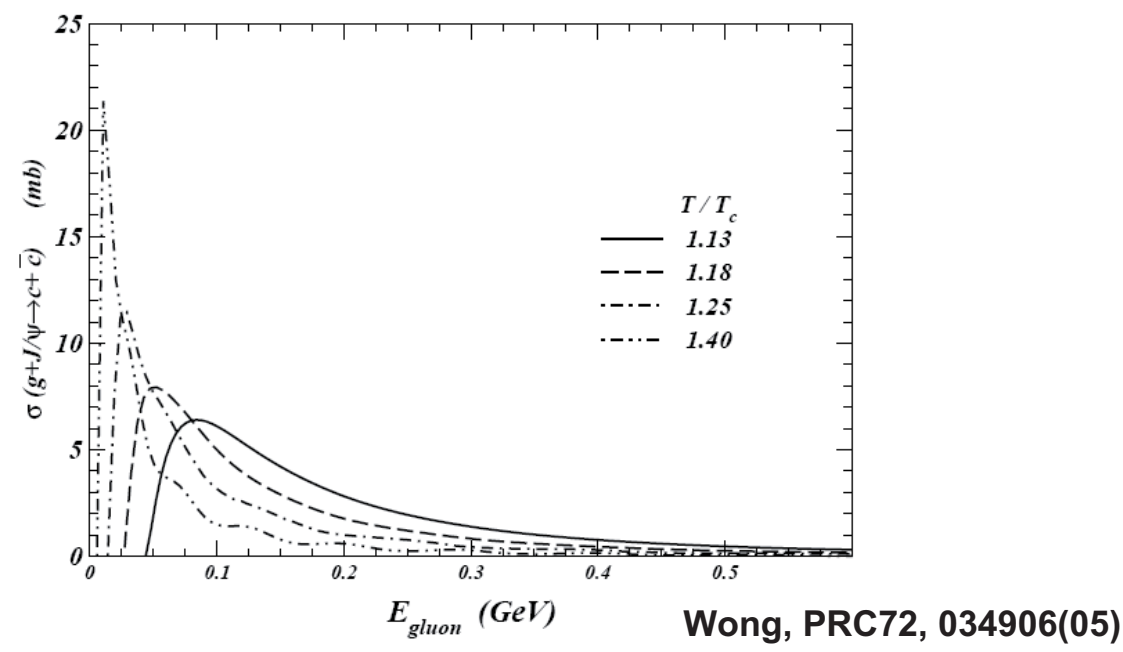




\section{Inverse reaction}

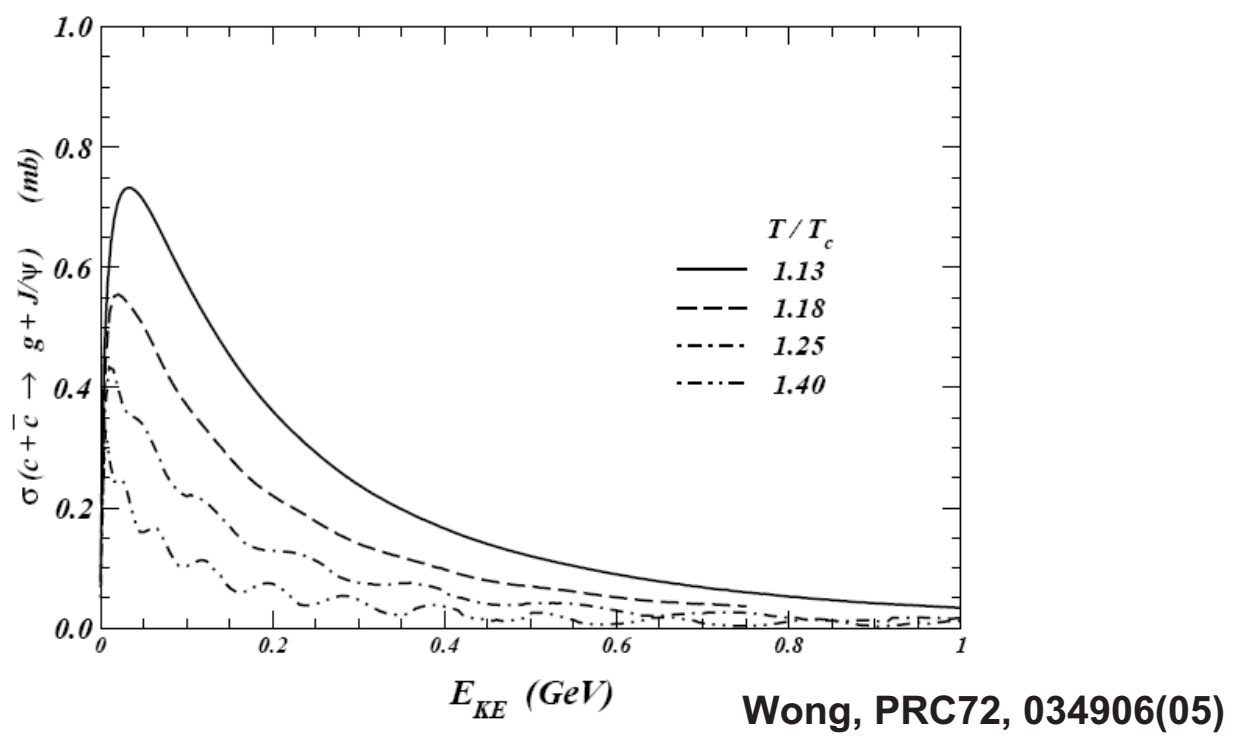

